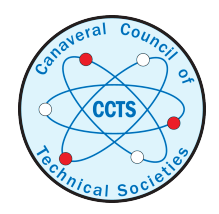

Dec 7th, 9:30 AM

\title{
Multi-Use Facilities - Repurposing Facilities Infrastructure to Support the Multi-customer Environment
}

\author{
Dave Hertzler \\ Engineers, BRPH Architects, Constructors \\ Alan T. DeLuna \\ Engineers, BRPH Architects, Constructors
}

Follow this and additional works at: https://commons.erau.edu/space-congress-proceedings

\section{Scholarly Commons Citation}

Hertzler, Dave and DeLuna, Alan T., "Multi-Use Facilities - Repurposing Facilities Infrastructure to Support the Multi-customer Environment" (2012). The Space Congress ${ }^{\circledR}$ Proceedings. 14.

https://commons.erau.edu/space-congress-proceedings/proceedings-2012-42nd/december-07-2012/14

This Event is brought to you for free and open access by the Conferences at Scholarly Commons. It has been accepted for inclusion in The Space Congress ${ }^{\circledR}$ Proceedings by an authorized administrator of Scholarly Commons. For more information, please contact commons@erau.edu.

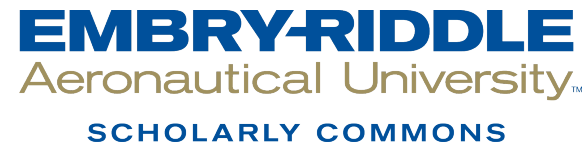




\section{Multi-Use Facilities - Repurposing Facilities Infrastructure to Support the Multi-customer Environment}

Dave Hertzler

Senior Project Manager

Alan T. DeLuna

Aerospace Consultant

BRPH ARCHITECTS / ENGINEERS / CONSTRUCTORS, Melbourne, Florida, USA 32940 


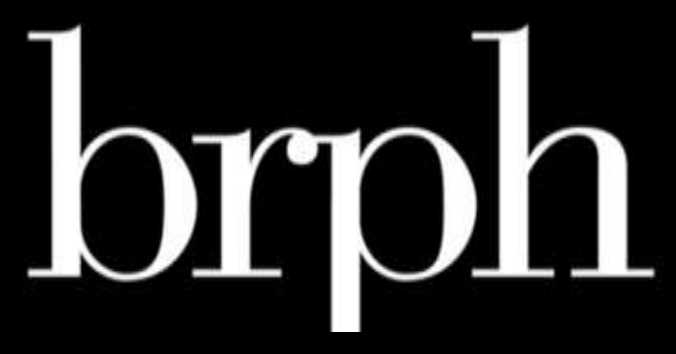




\section{Project Locations}

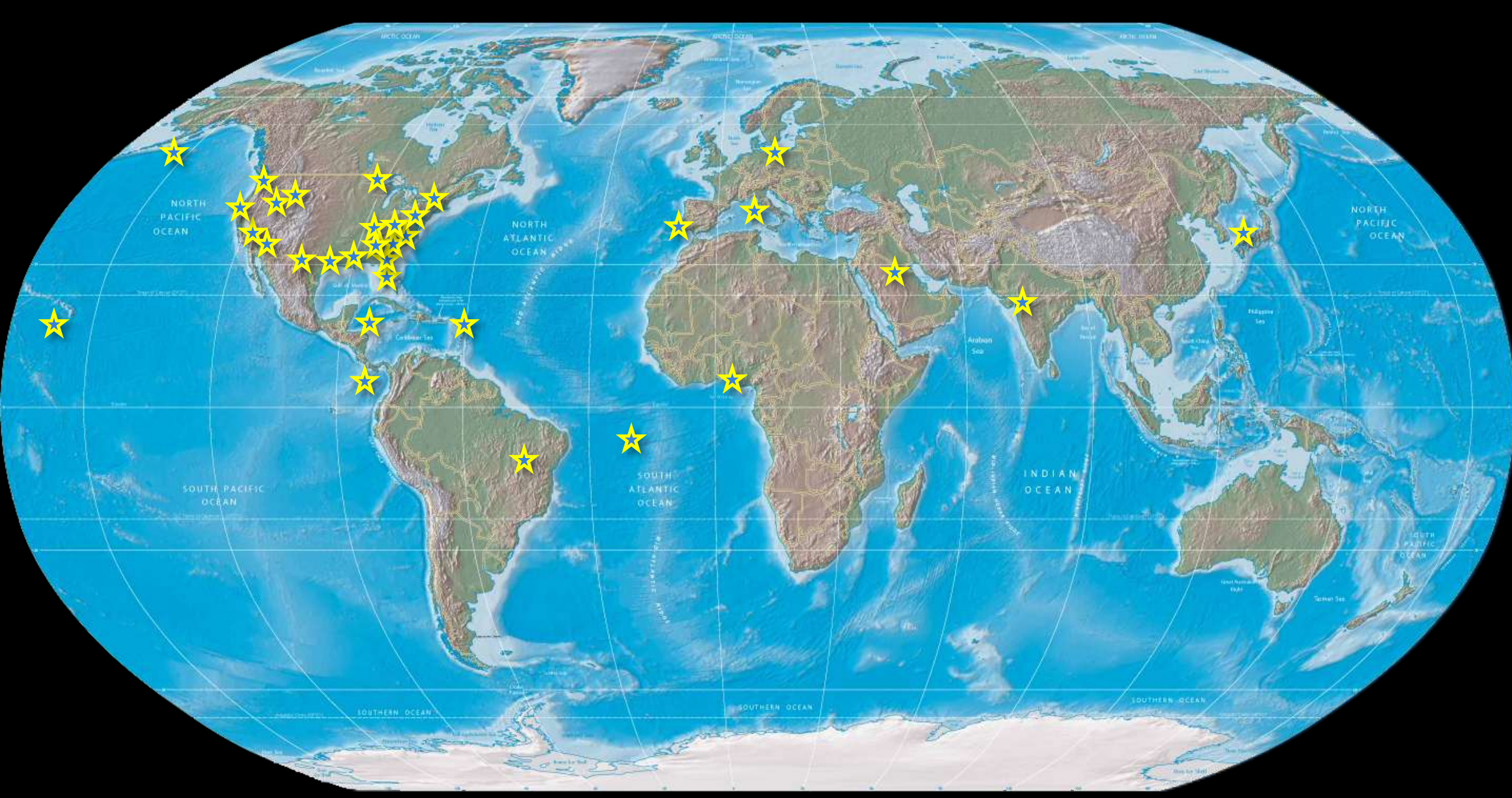

- Extensive use of Local Resources

- World Wide Experience in Partnering 


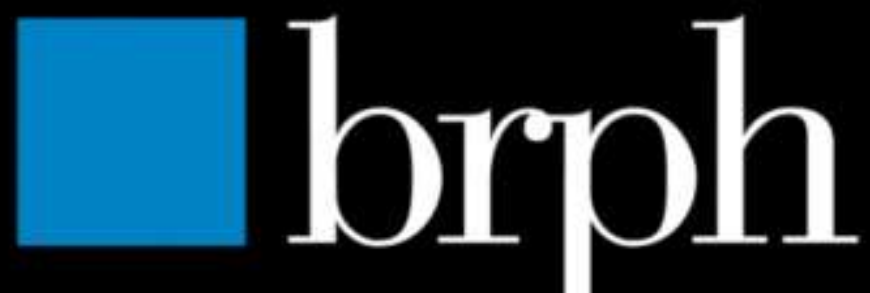

\section{Years of Experience at CCAFS and KSC}

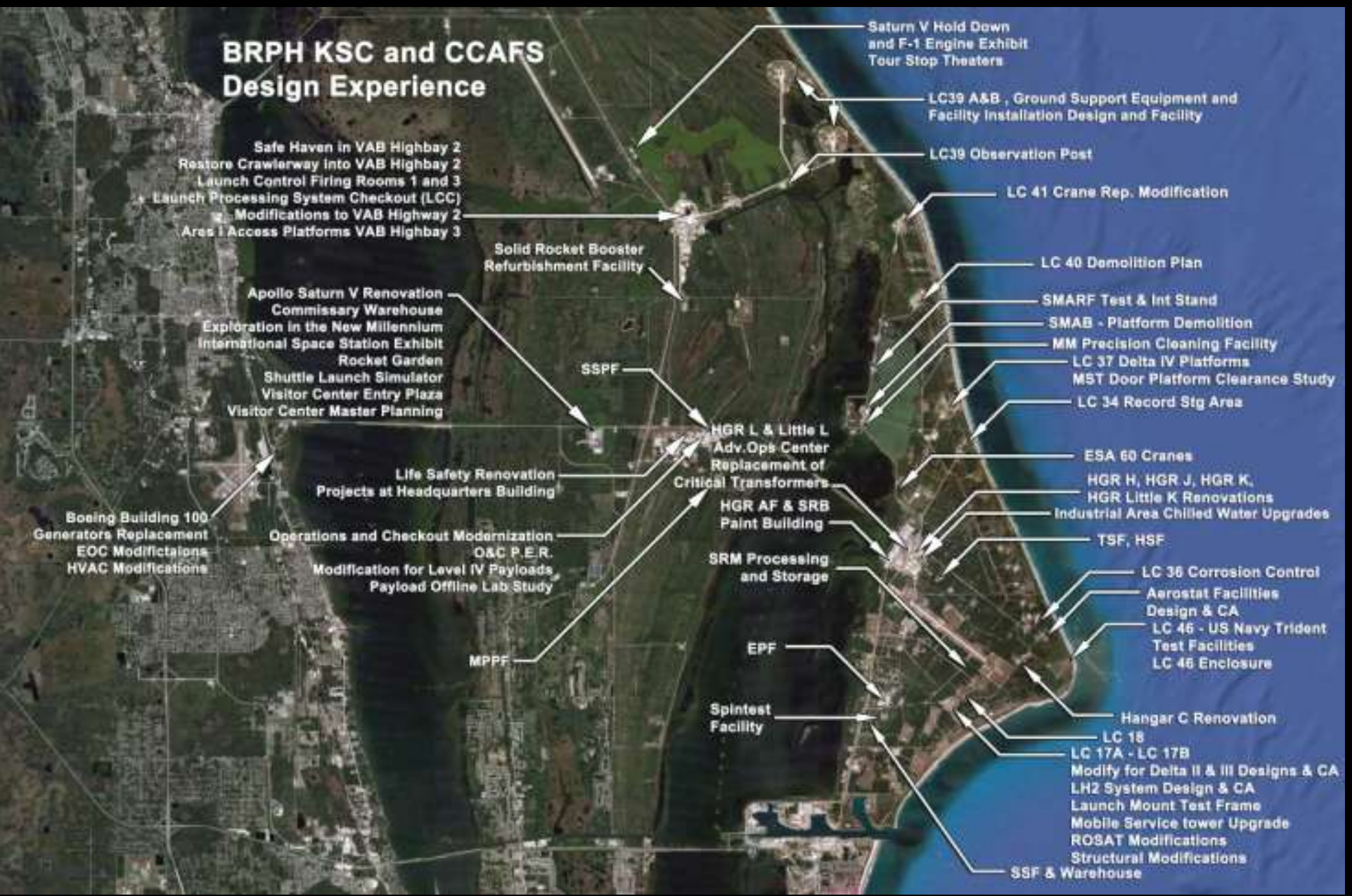




\section{Summary}

- Repurposing existing facilities to support the multi-customer has advantages for the facility owner and the eventual tenant.

- Determining the facility changes necessary is reasonably straightforward although it can be a significant amount of work. 


\section{Old Yankee Stadium}

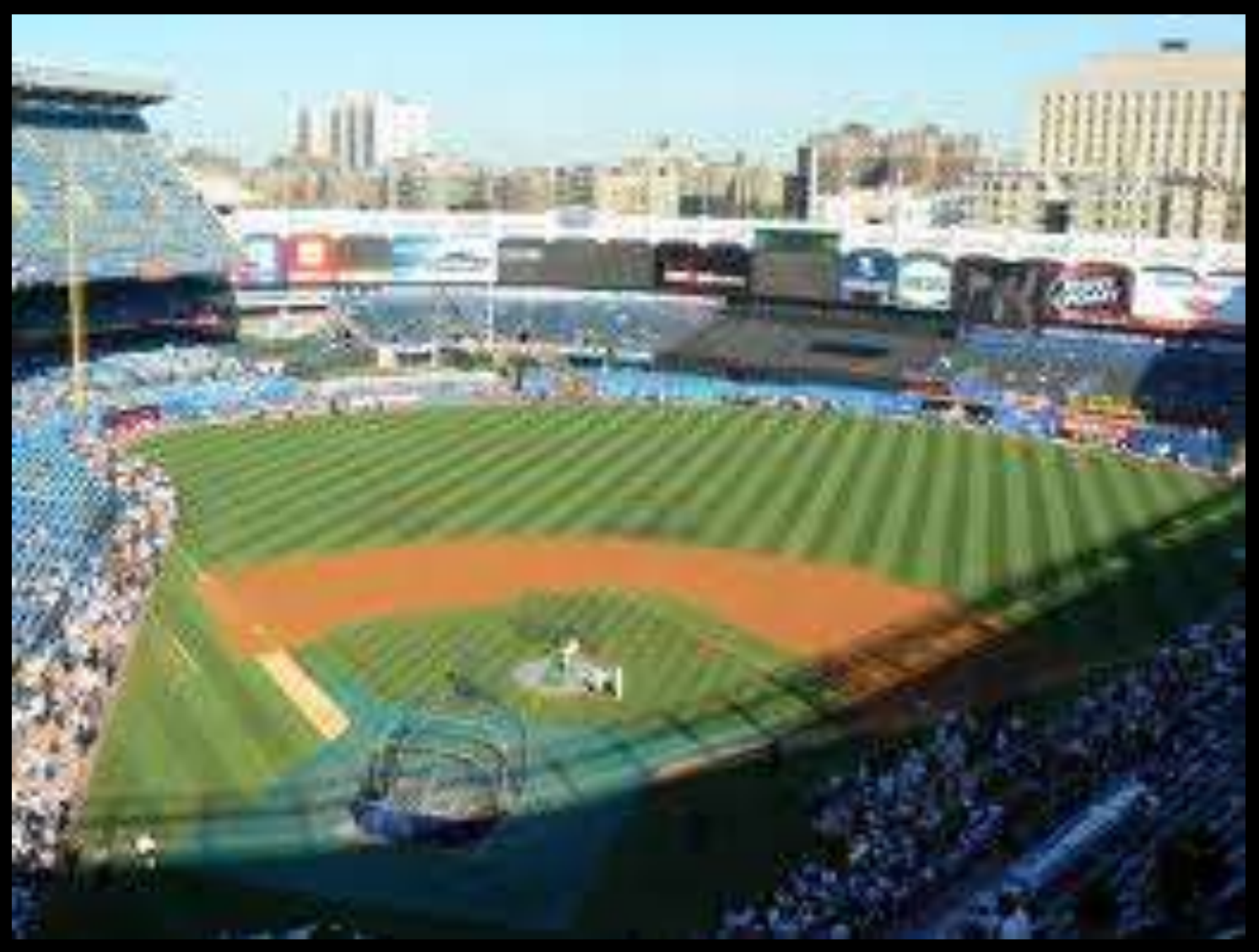




\section{New Yankee Stadium}

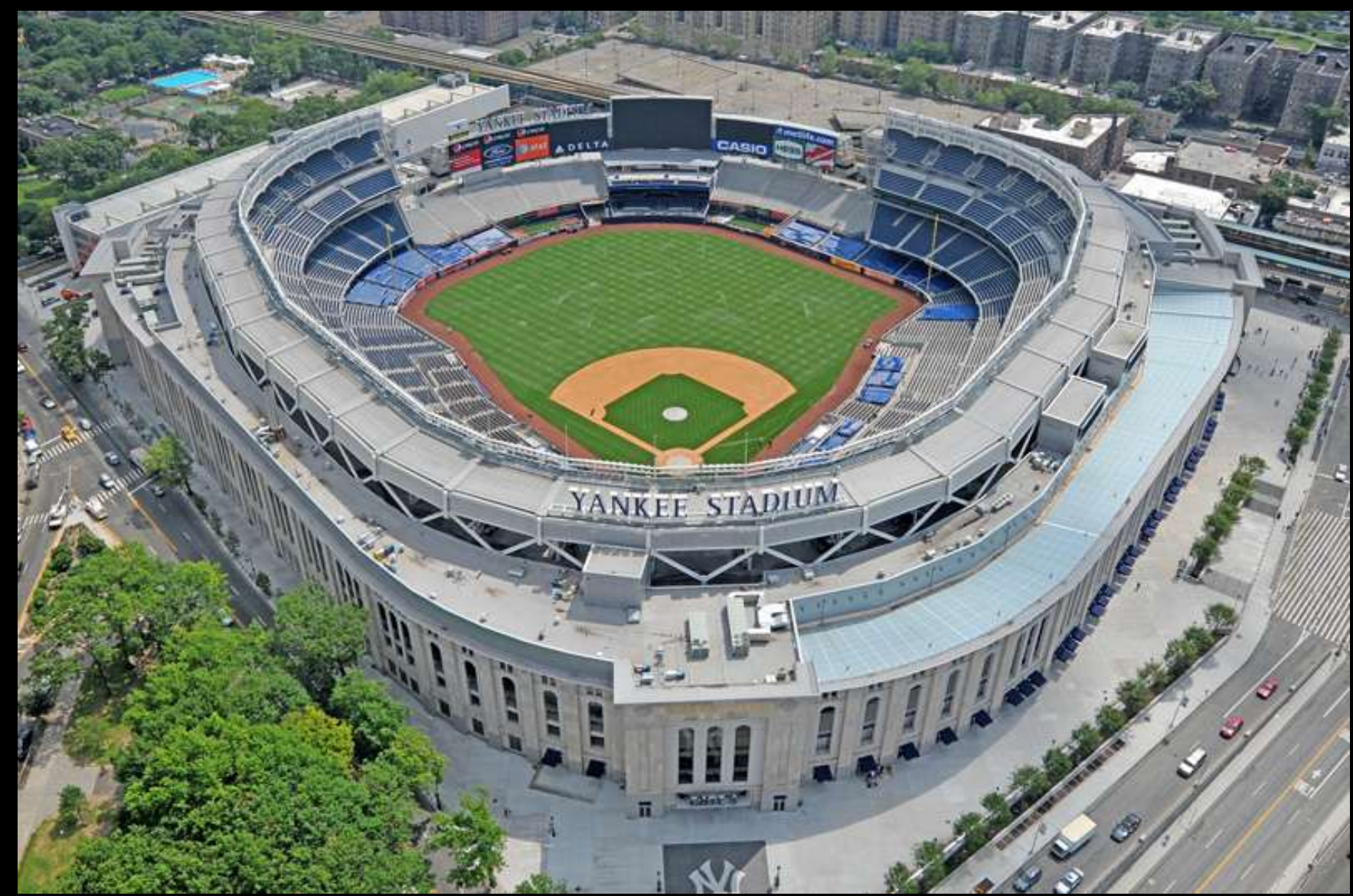




\section{It Is Multiuse}

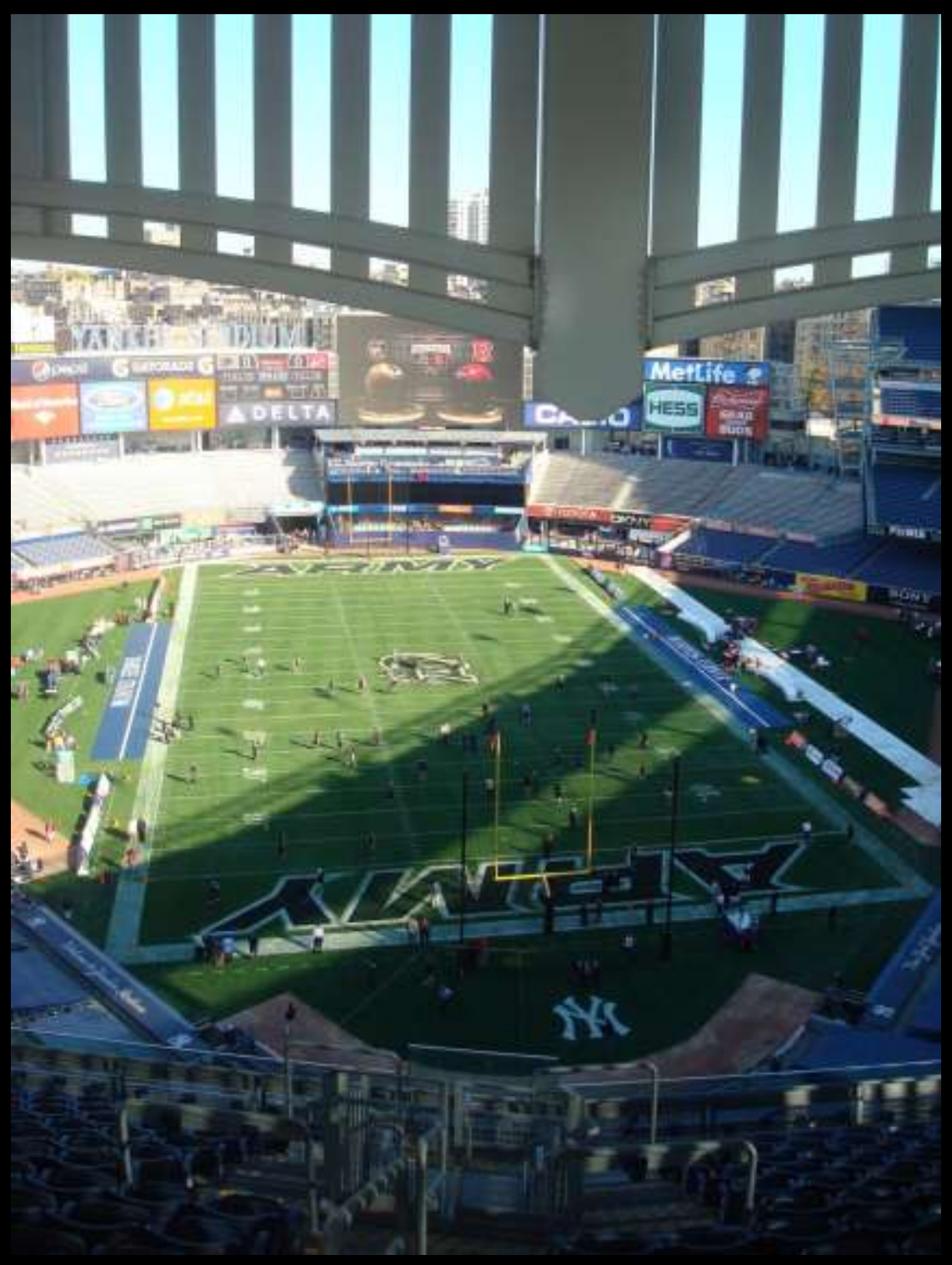




\section{Better Multi-use}

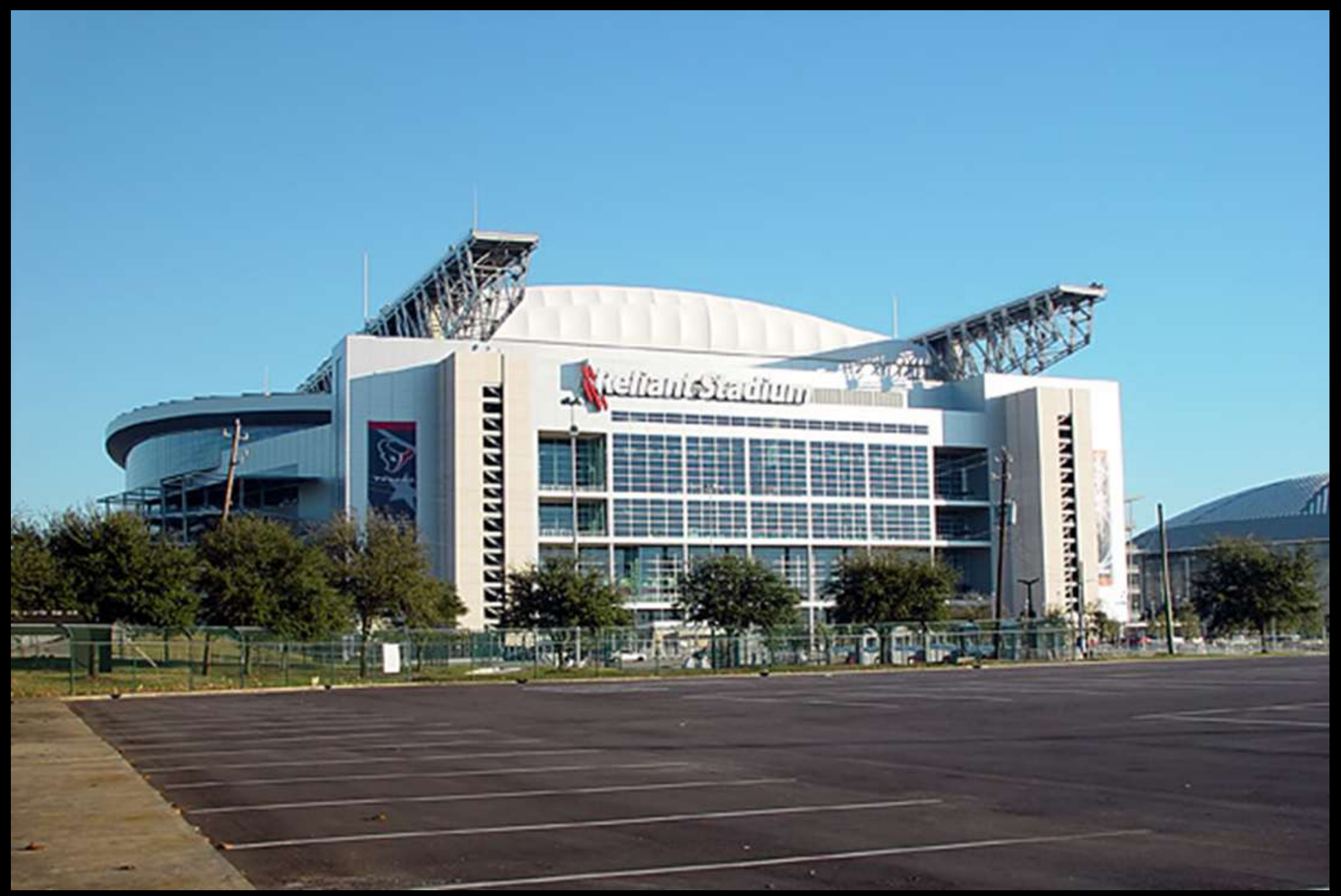

Photohome.com 


\section{Better Multi-use}

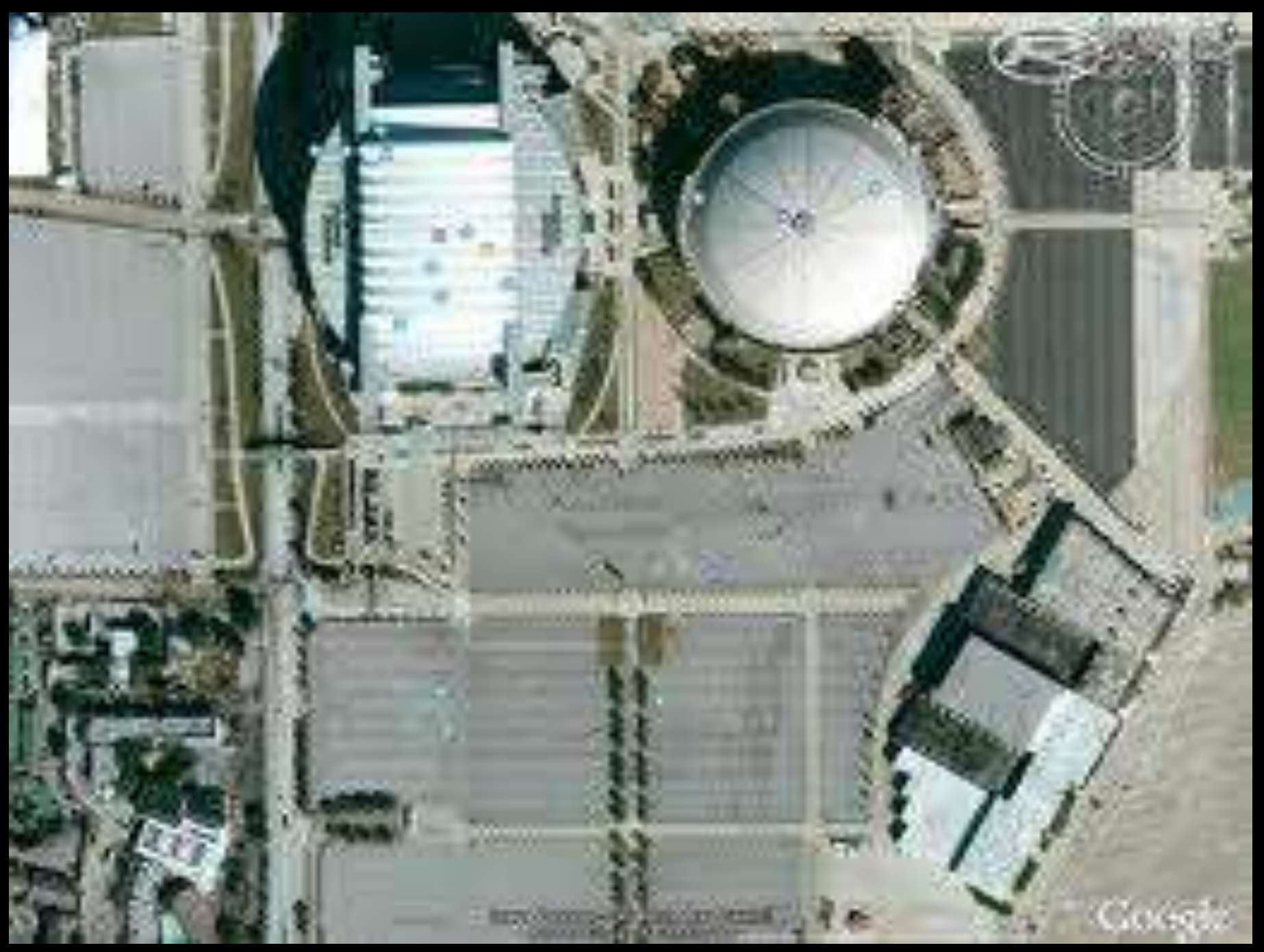




\section{Better Multi-use}

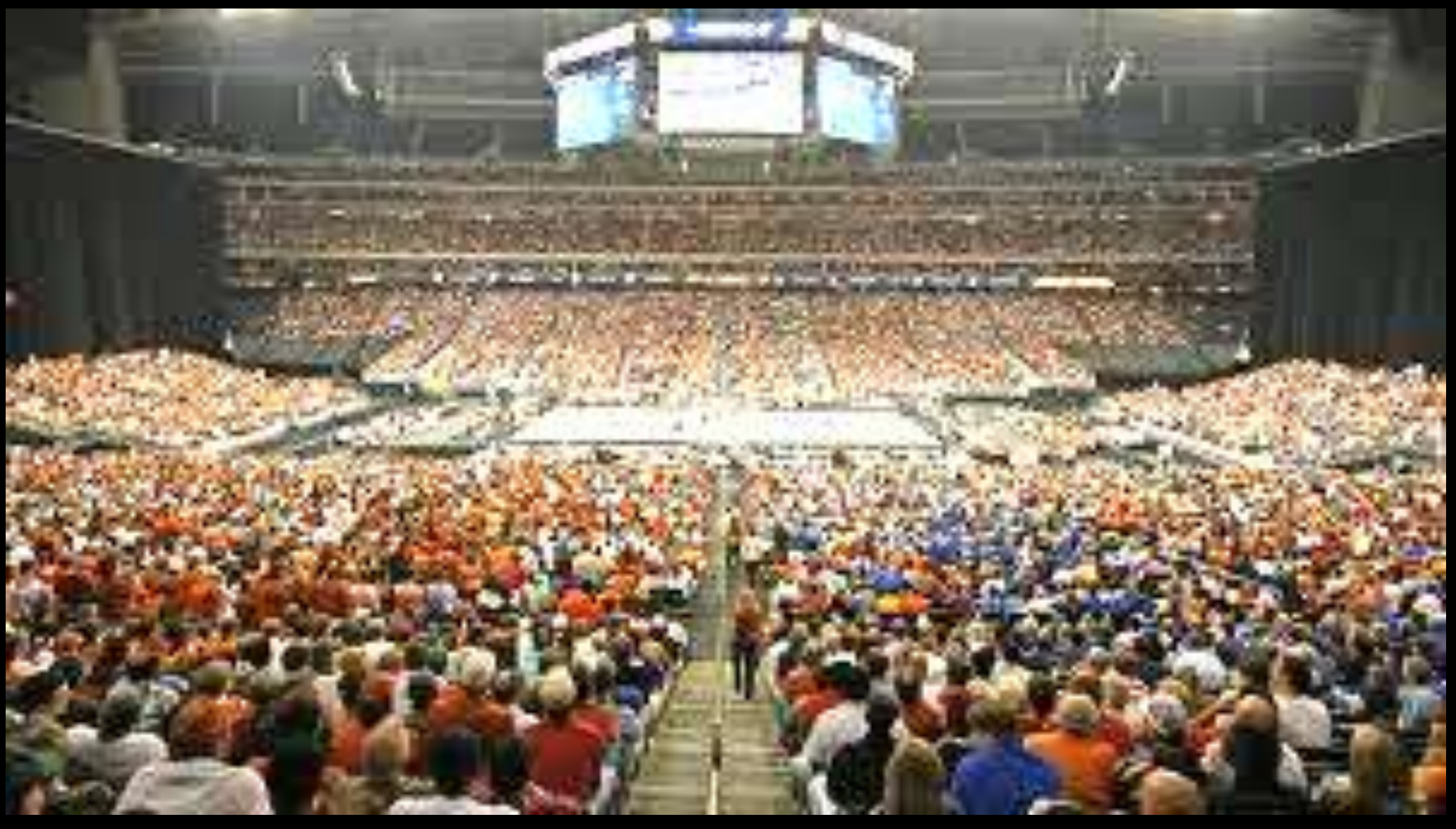




\section{Better Multi-use}

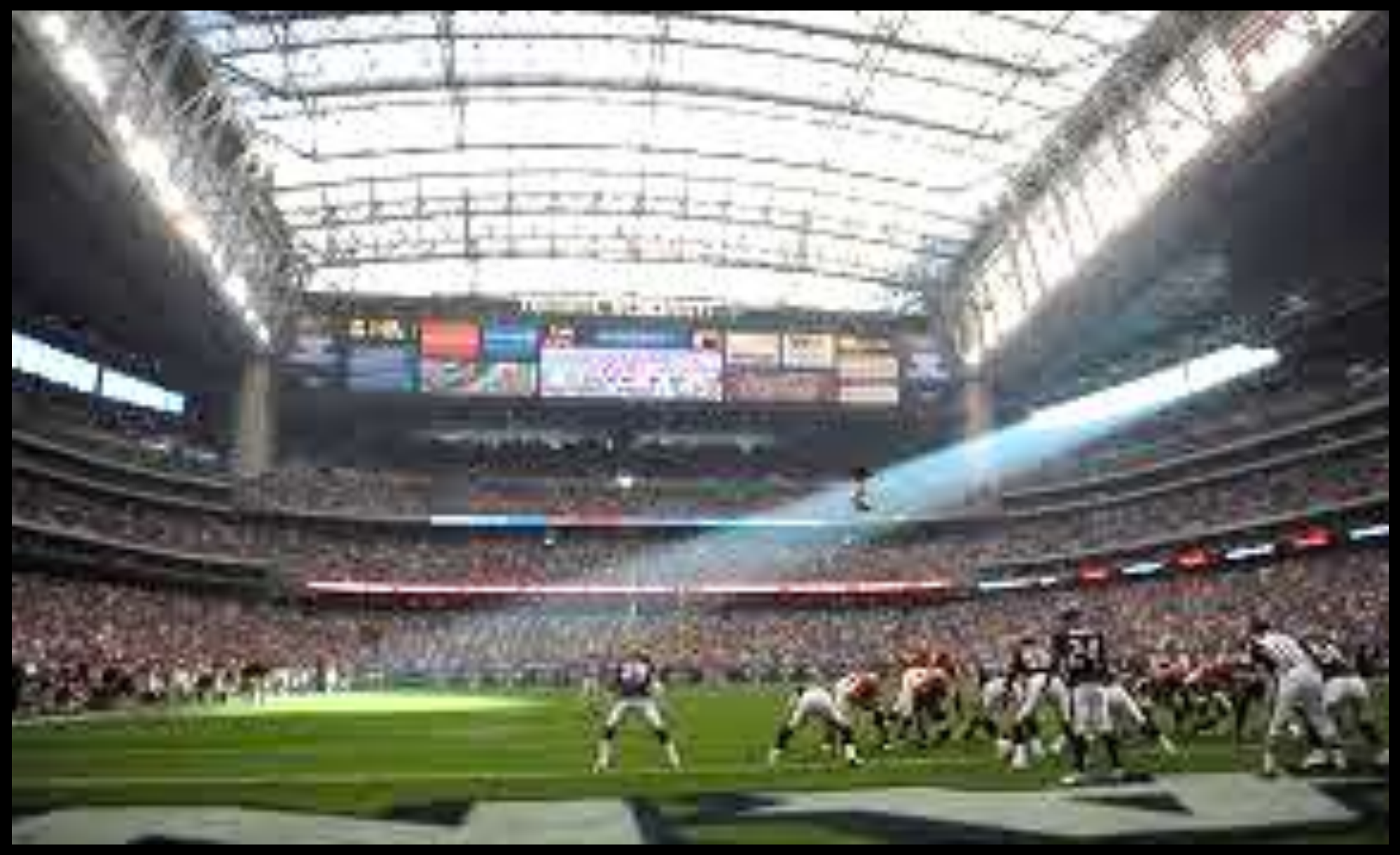




\section{KSC has a history of multi-use - sort of....}




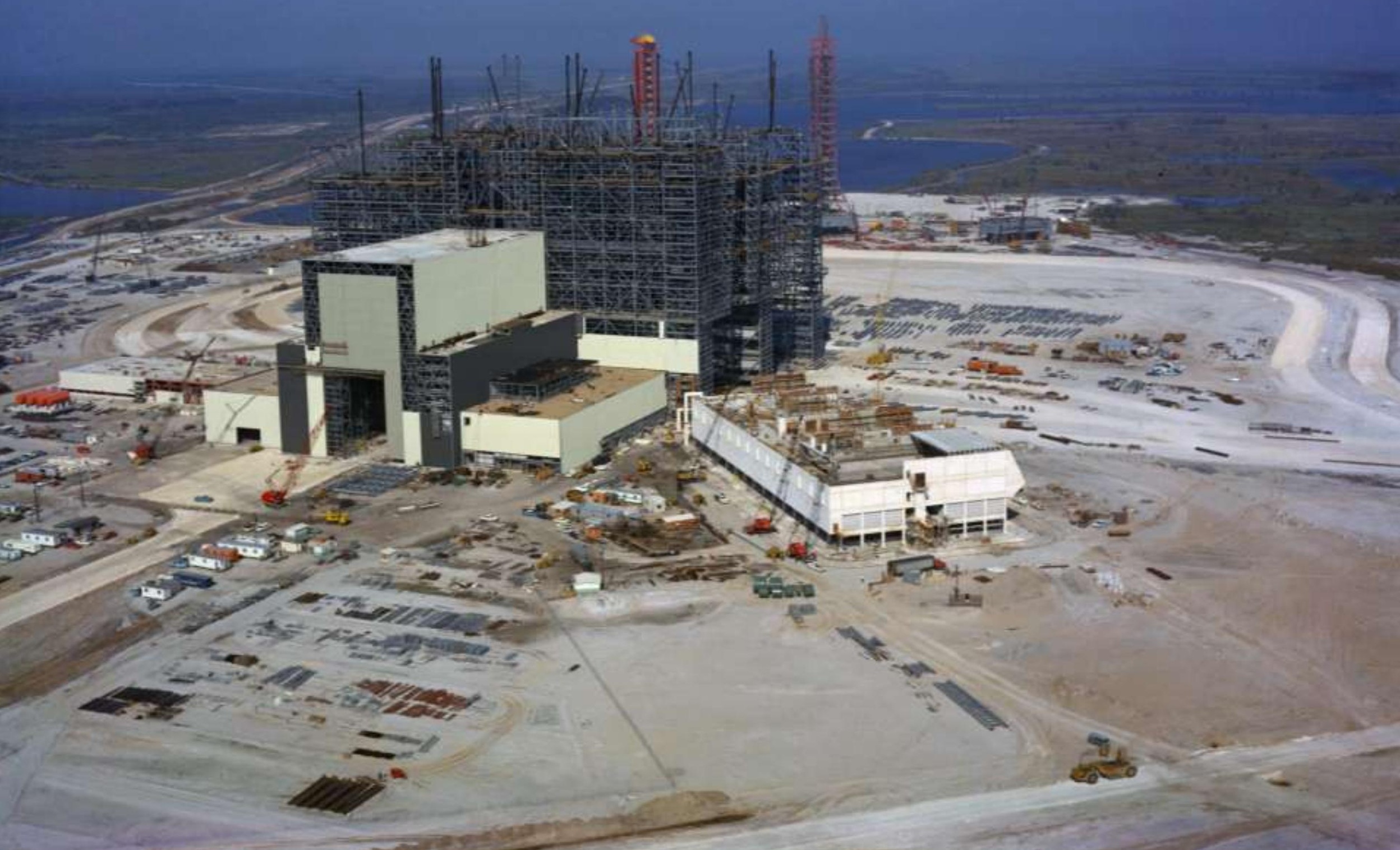



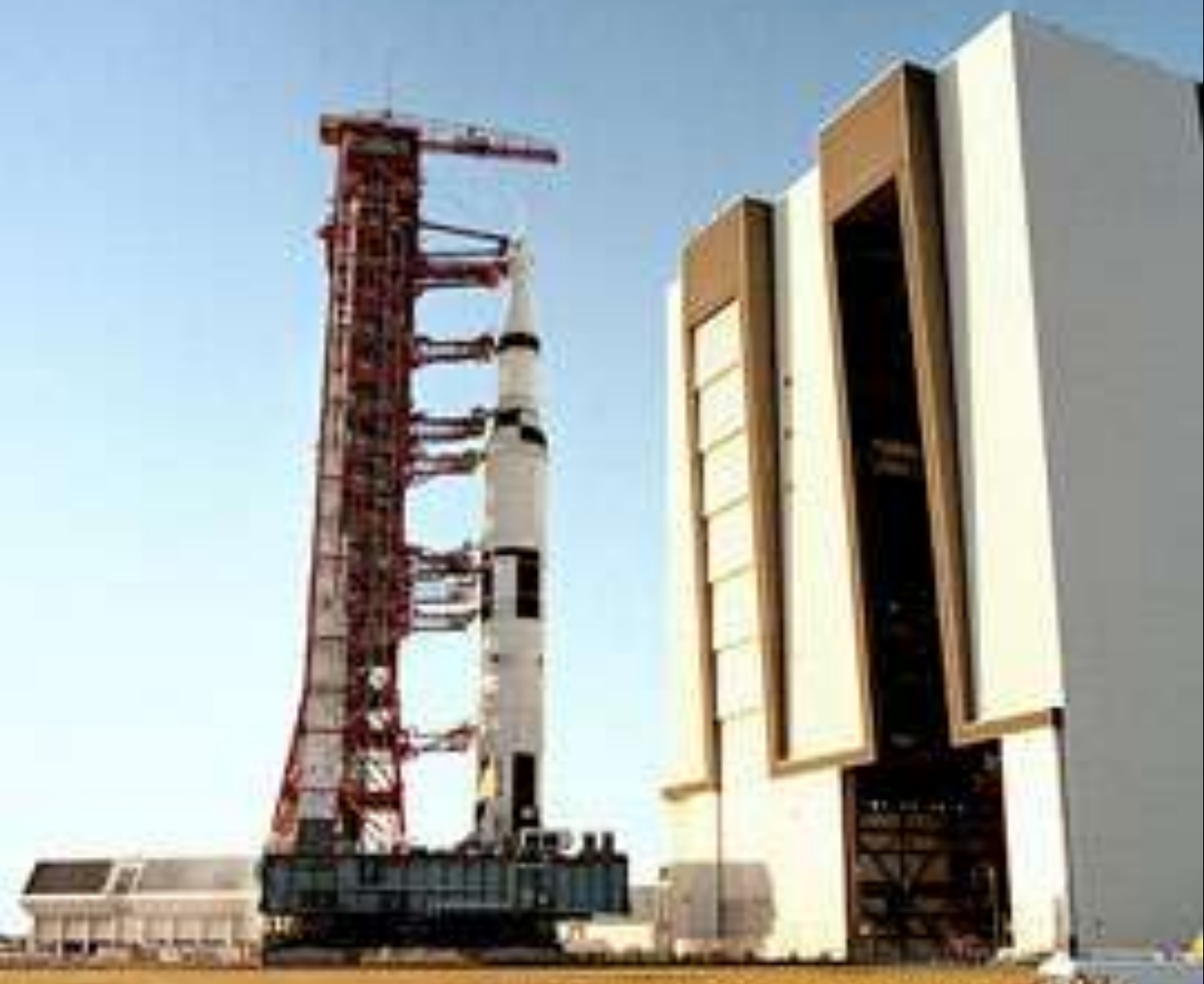

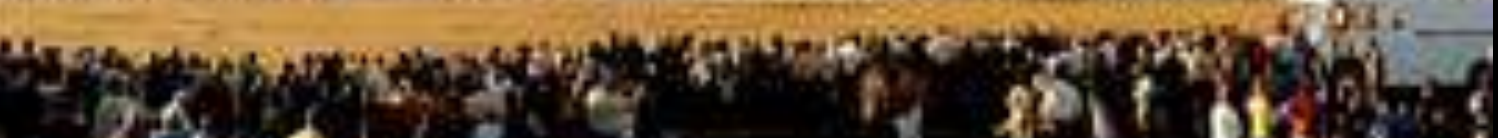

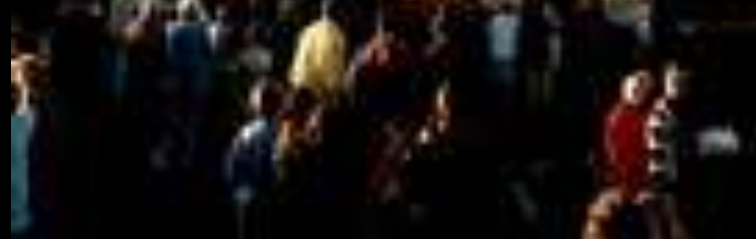

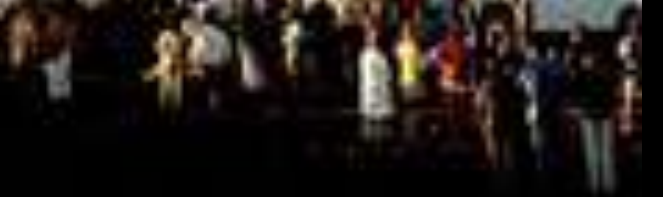




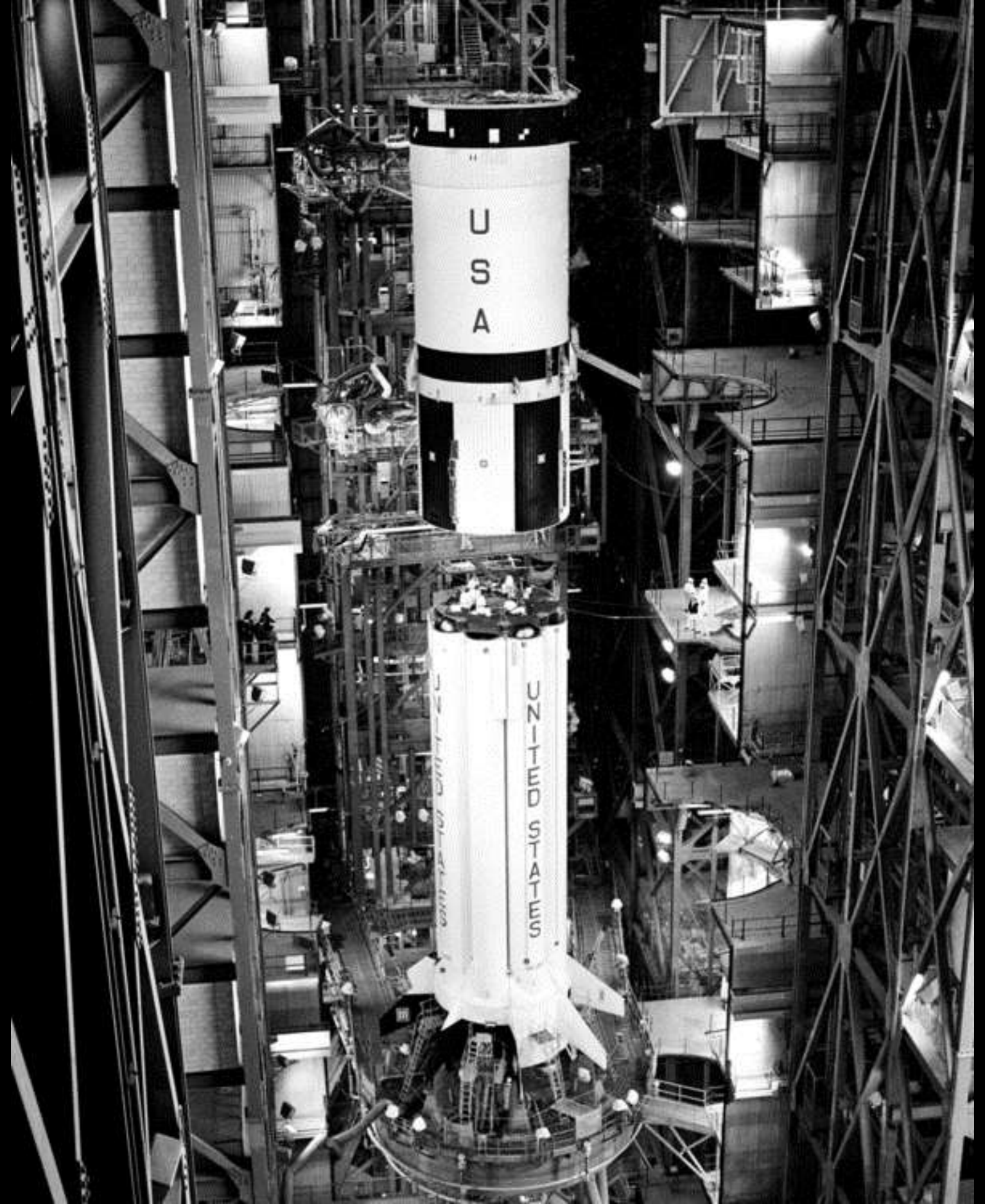




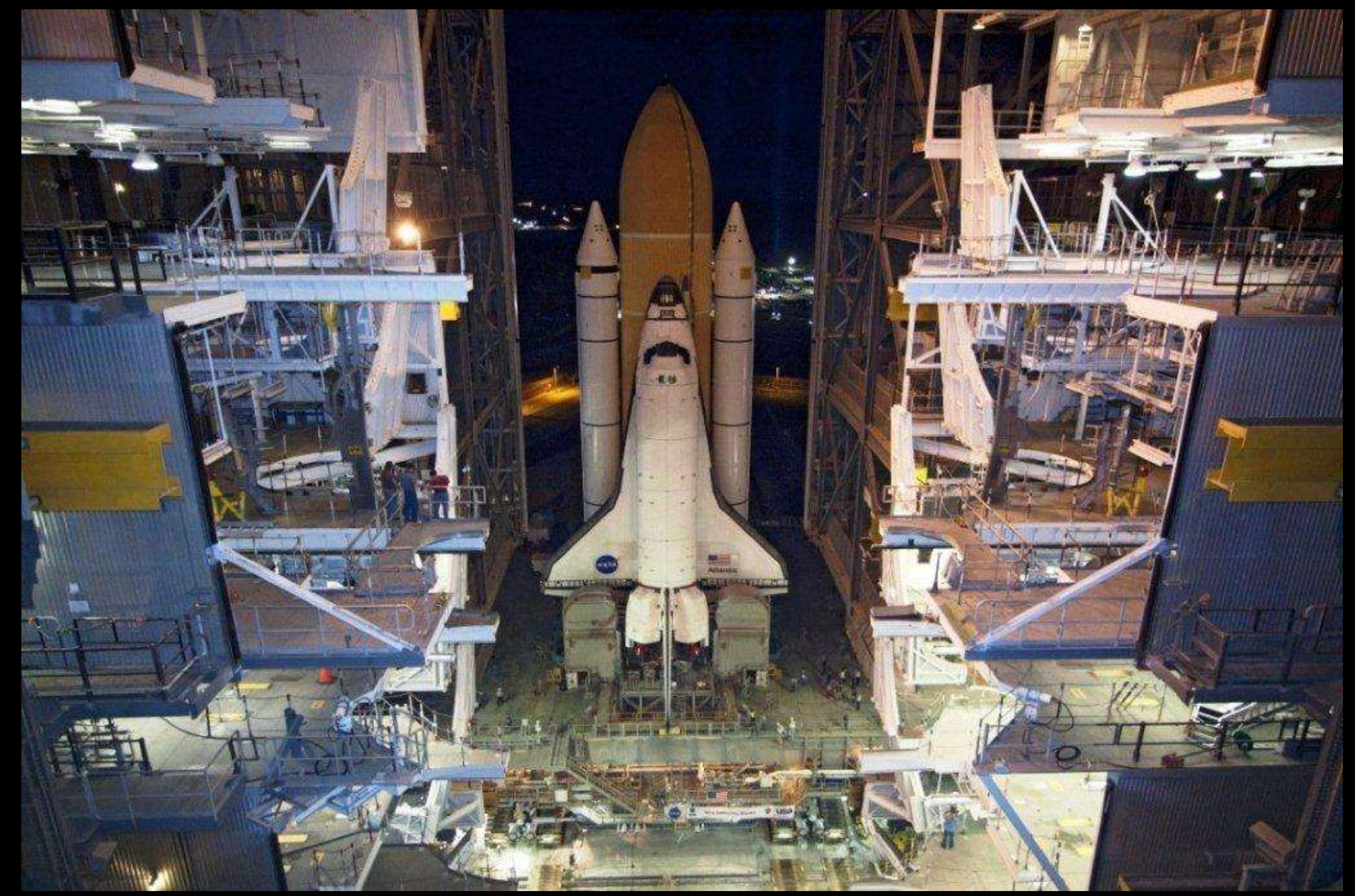




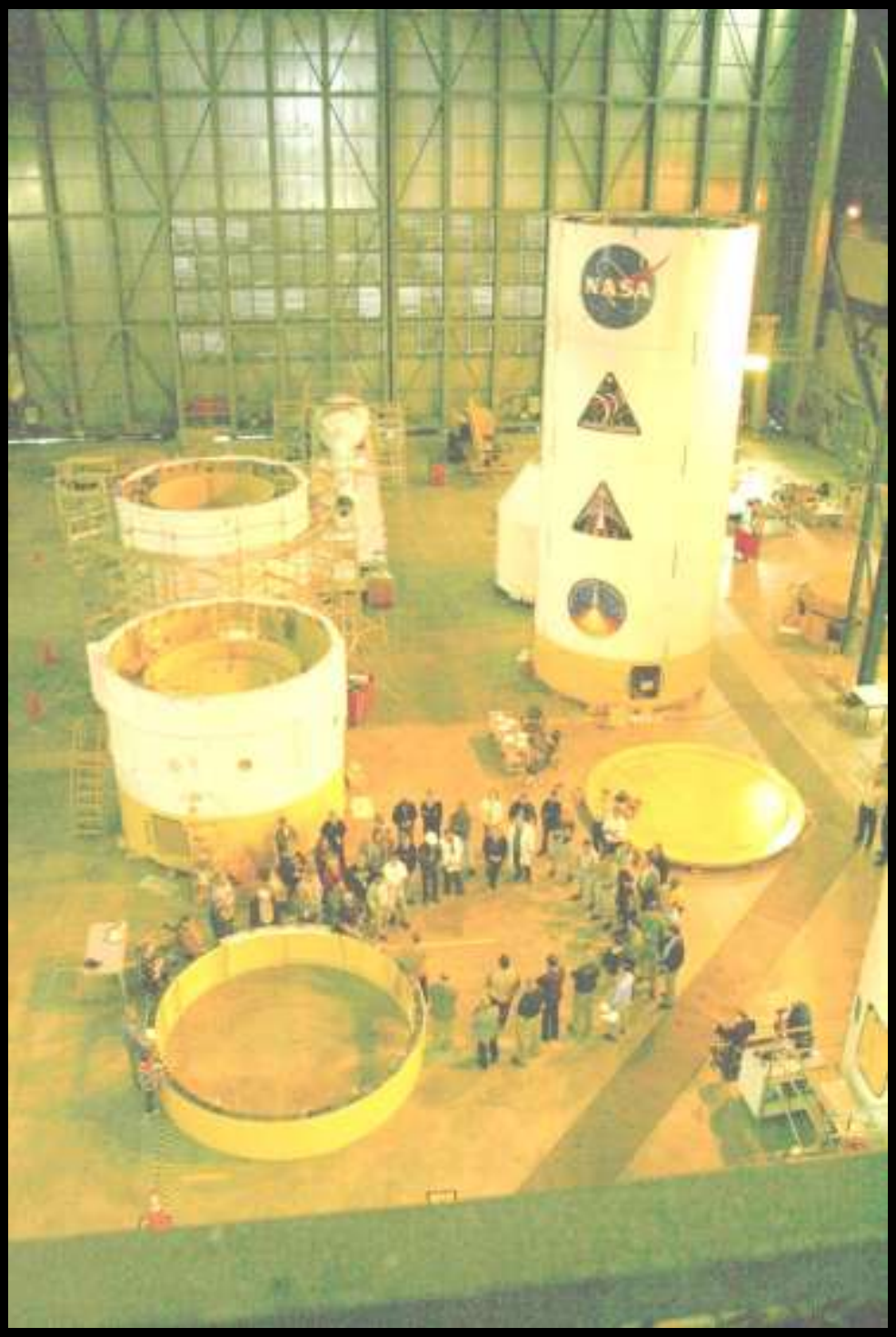




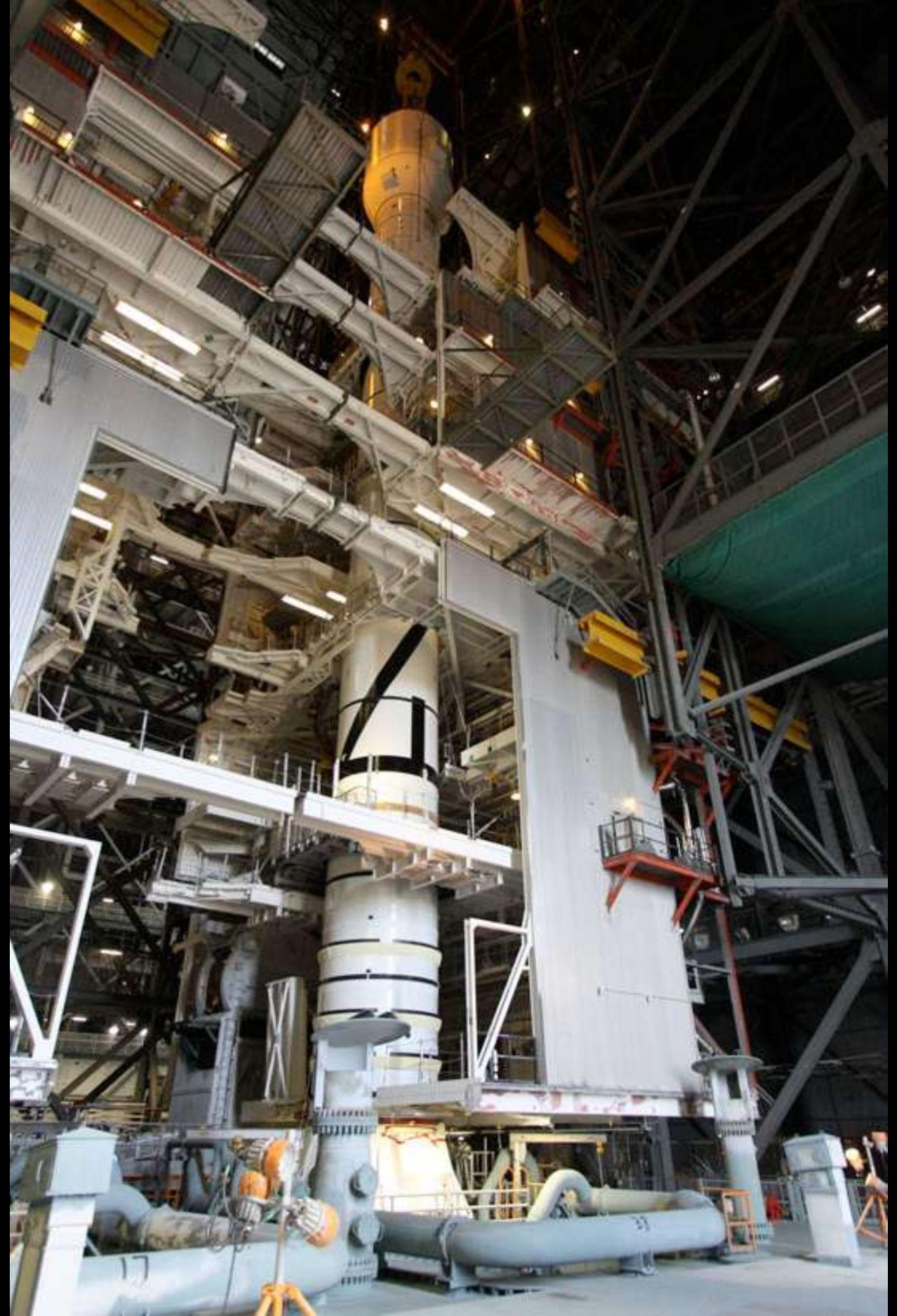




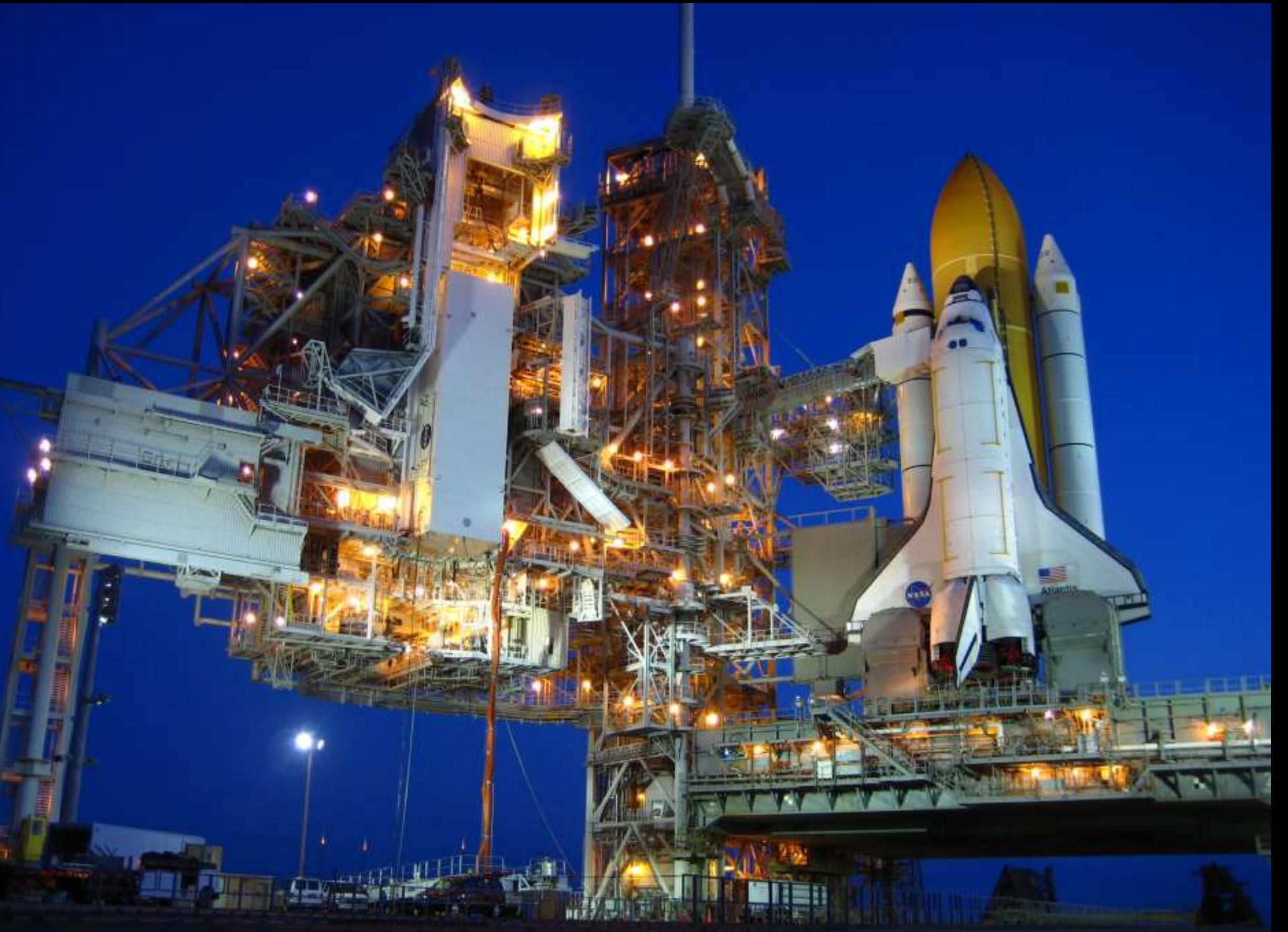




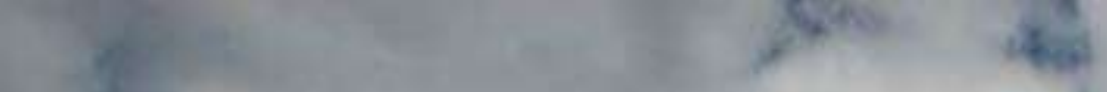




\section{Where in the world is multi-use?}

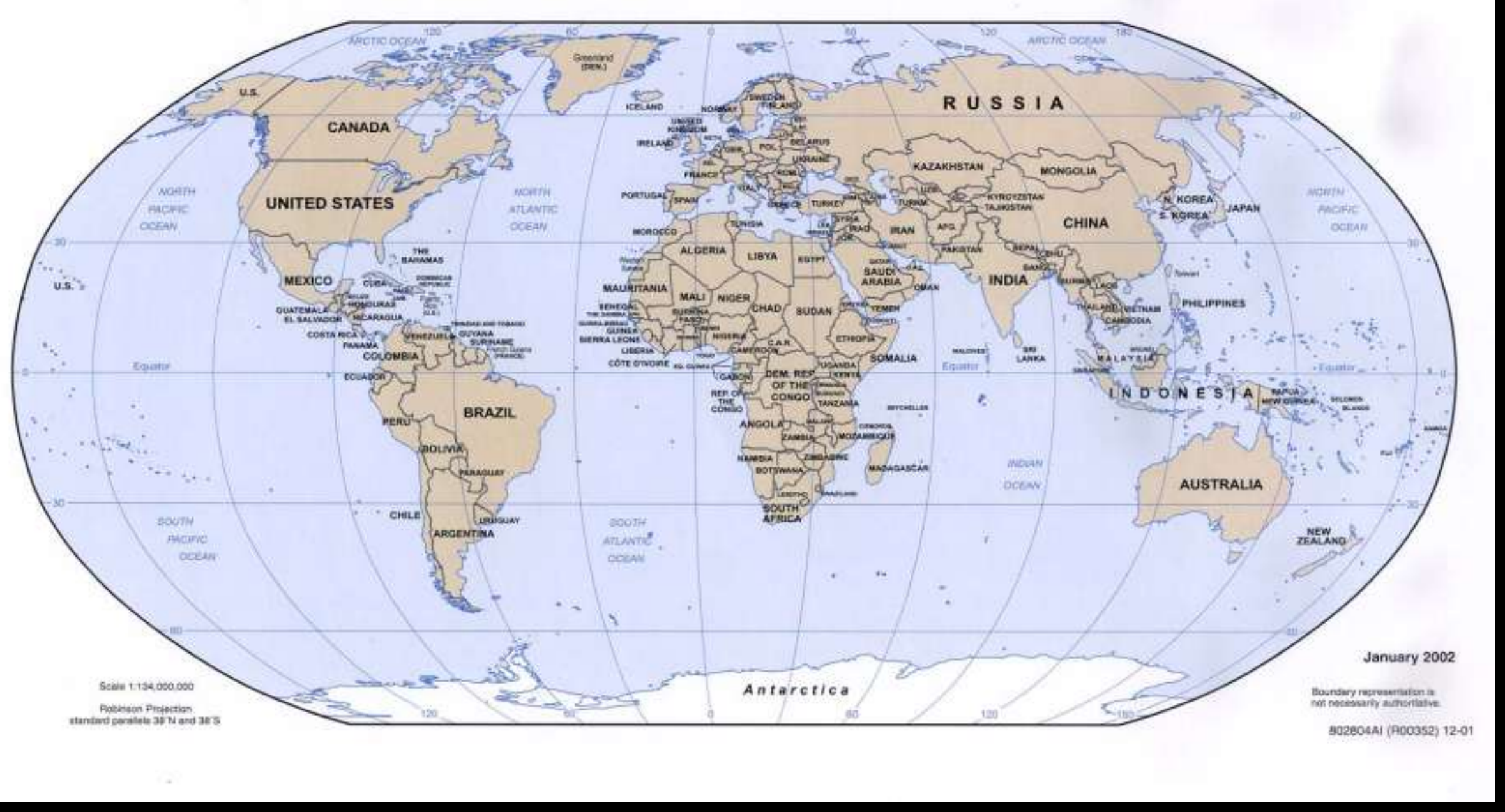




\section{How do we get there?}

- Understand why multi-use is desirable

- Decide the best usage categories

- Identify target tenants

- Perform an operator needs assessment

- Analyze the facility's capabilities, capacity, and restrictions

- Perform a gap analysis

- Develop Operational Concepts

- Map modifications to concepts

- Make decisions 


\section{Steps In The Process}

- Understand why multi-ı ' 9 _esirable

- Decide the best usas

- Identify target te ${ }^{0}$

- Perform an or $e^{\text {d }}$. needs assessment

- Analyze thr $\&^{5}$. .cy's capabilities, capacity, and rest" 1 is

- Perfc $\boldsymbol{N}^{\boldsymbol{B}}$ sap analysis

- Develo, Operational Concepts

- Map modifications to concepts

- Make decisions 


\section{We have a course Where does it take us}

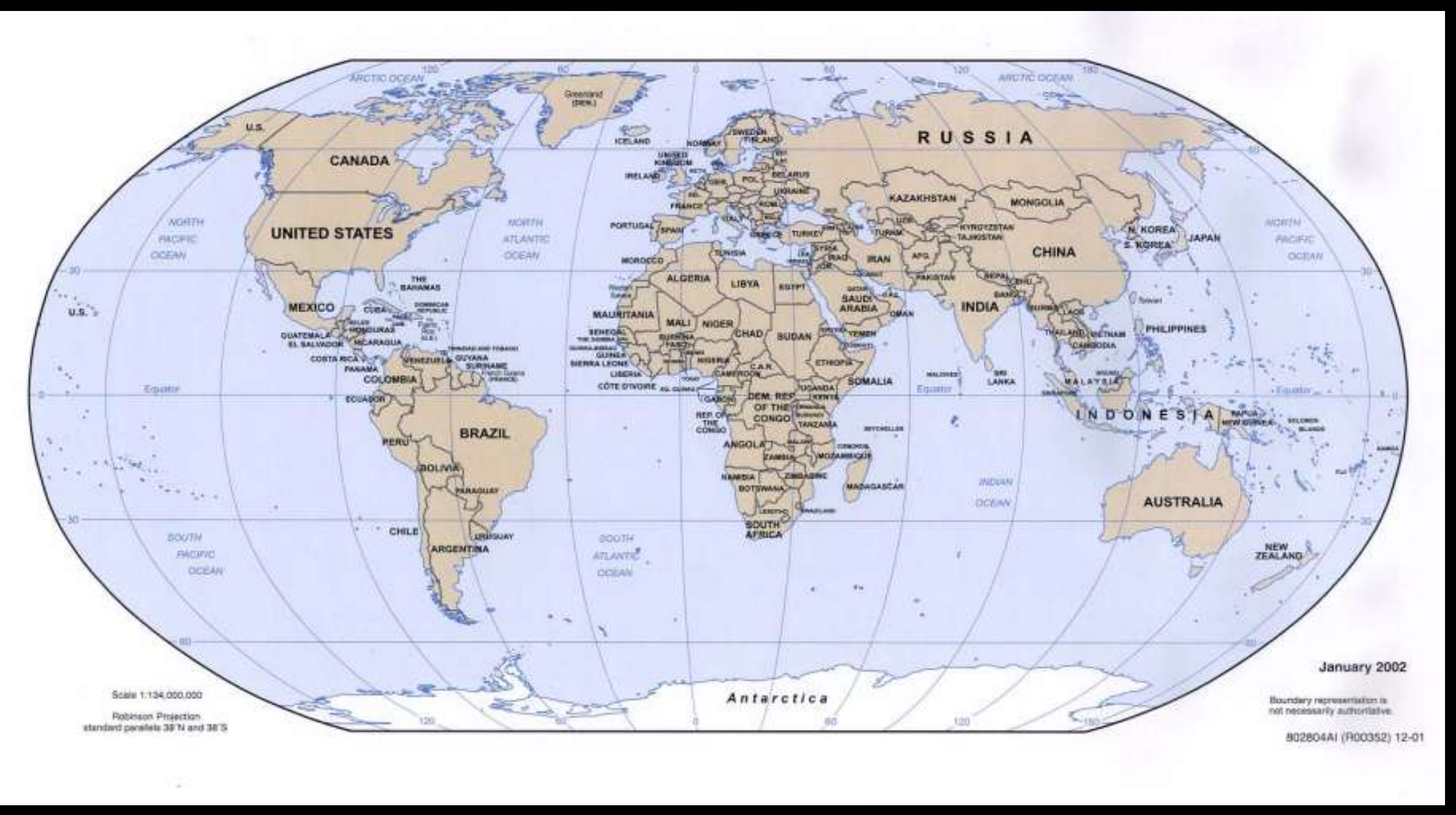




\section{Understand Why Multi-use Is Desirable}




\section{Decide The Best Usage Categories}
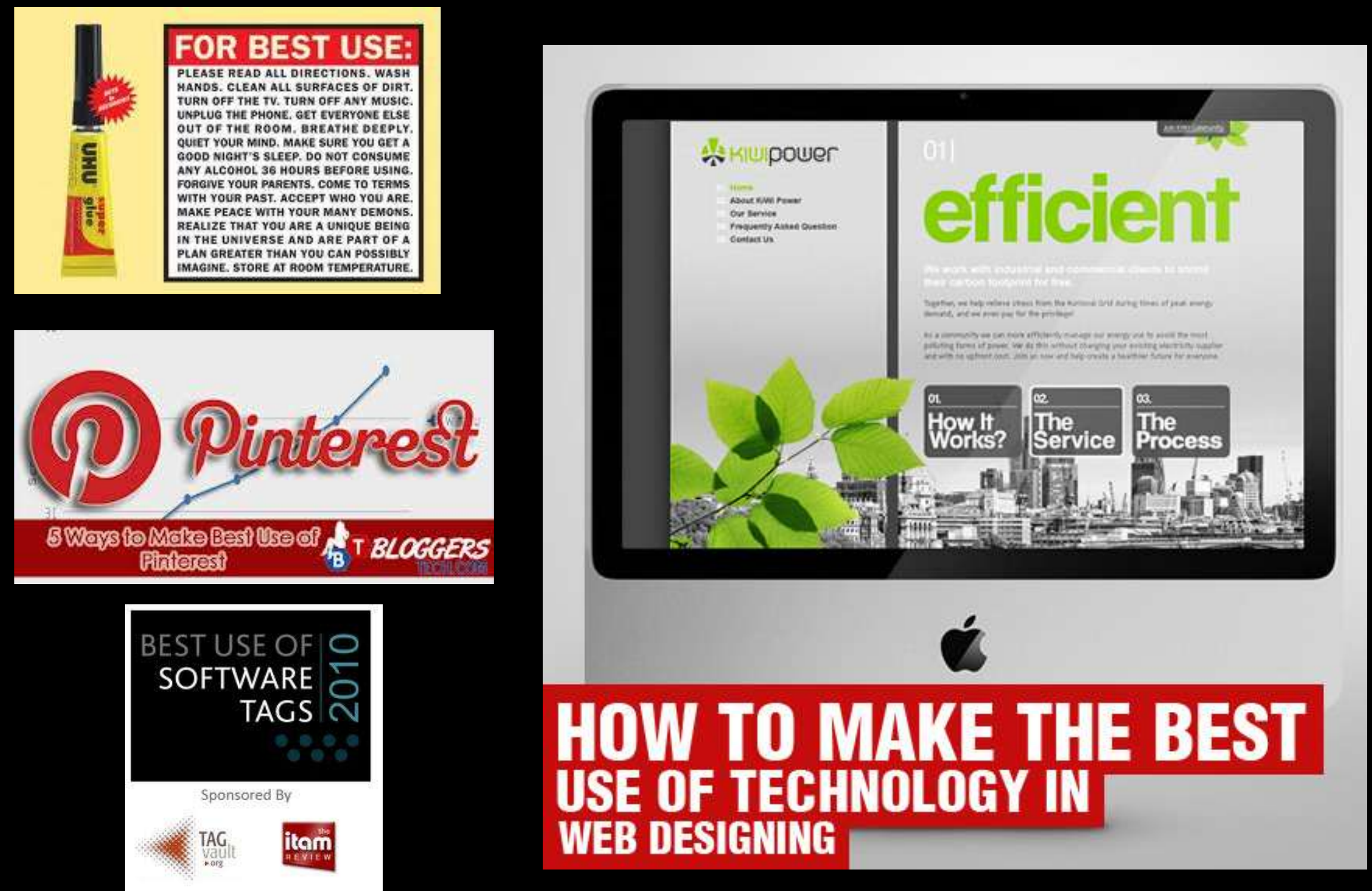


\section{Decide The Best Usage Categories}

\begin{tabular}{|c|c|c|c|c|c|c|}
\hline $\begin{array}{l}\text { Launch } \\
\text { Vehicle }\end{array}$ & $\begin{array}{l}\text { Booster and } \\
\text { Upper Stage }\end{array}$ & $\begin{array}{l}\text { Satellite and } \\
\text { Spacecraft }\end{array}$ & Launch & Hazards & $\begin{array}{l}\text { Operational } \\
\text { restrictions }\end{array}$ & Other \\
\hline $\begin{array}{l}\text { Large } \\
\text { Vehicle }\end{array}$ & Solid & Manufacturing & Horizontal & Industrial & $\begin{array}{c}\text { Parallel } \\
\text { Hazardous } \\
\text { Operations }\end{array}$ & $\begin{array}{l}\text { Offline } \\
\text { Shops }\end{array}$ \\
\hline $\begin{array}{c}\text { Small } \\
\text { Vehicle }\end{array}$ & Liquid & Assembly & Vertical & Pyrotechnics & $\begin{array}{c}\text { Restricted } \\
\text { Tenant } \\
\text { Activity }\end{array}$ & $\begin{array}{l}\text { Offline } \\
\text { Labs }\end{array}$ \\
\hline Stacking & Manufacturing & Testing & $\begin{array}{c}\text { Small } \\
\text { vehicle }\end{array}$ & Hypergolics & $\begin{array}{l}\text { Man loading } \\
\text { restrictions }\end{array}$ & $\begin{array}{l}\text { Office } \\
\text { Space }\end{array}$ \\
\hline Testing & Assembly & $\begin{array}{c}\text { Fairing } \\
\text { Operations }\end{array}$ & $\begin{array}{l}\text { Large } \\
\text { Vehicle }\end{array}$ & Cryogenics & $\begin{array}{c}\text { Anchor } \\
\text { Tenant } \\
\text { Preferences }\end{array}$ & $\begin{array}{c}\text { Secure } \\
\text { Programs }\end{array}$ \\
\hline Integration & Testing & $\begin{array}{c}\text { Vehicle } \\
\text { Integration }\end{array}$ & & $\begin{array}{l}\text { Radiant } \\
\text { Energy }\end{array}$ & & \\
\hline
\end{tabular}




\section{Identify Target Tenants}

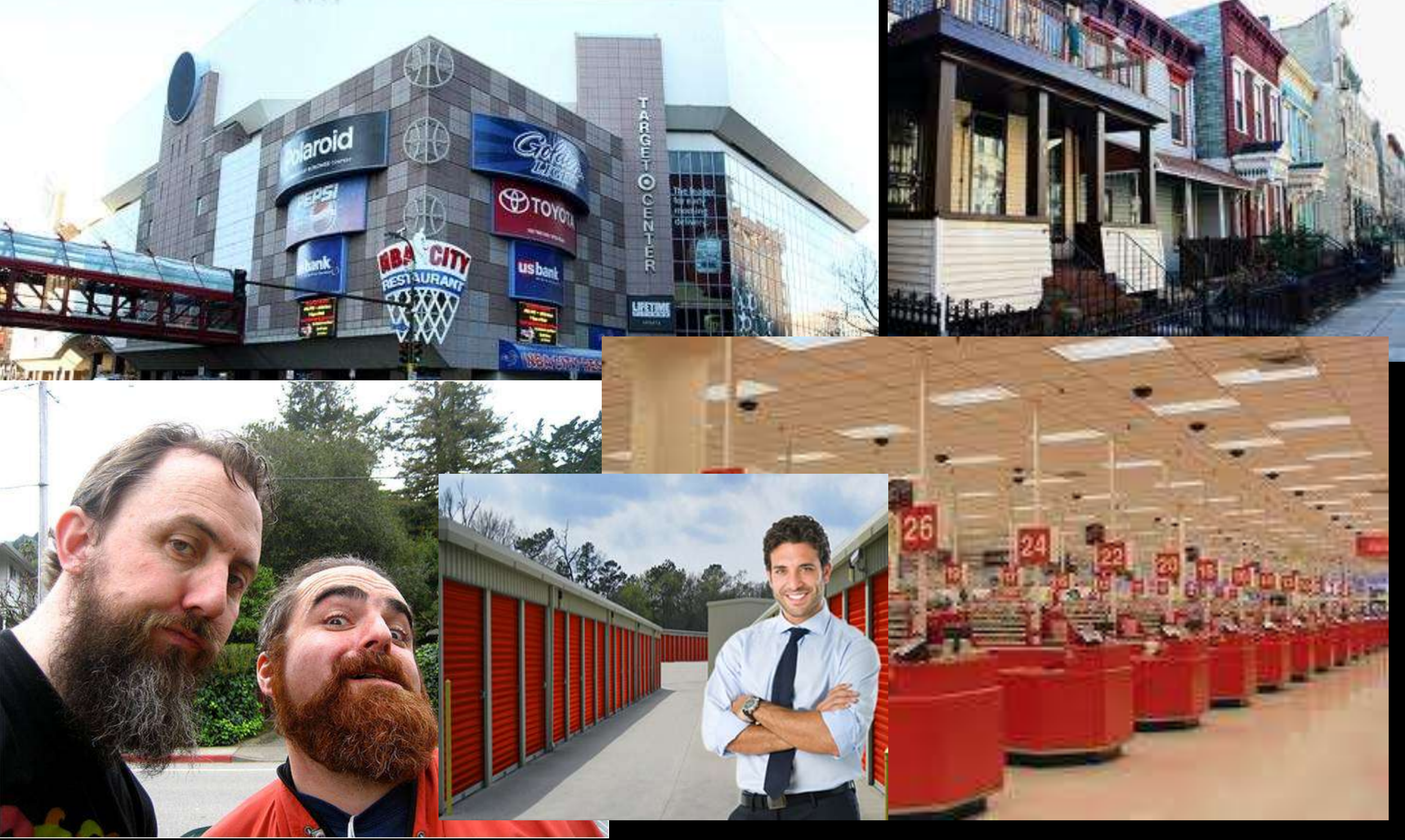




\section{Perform An Operator Needs Assessment}

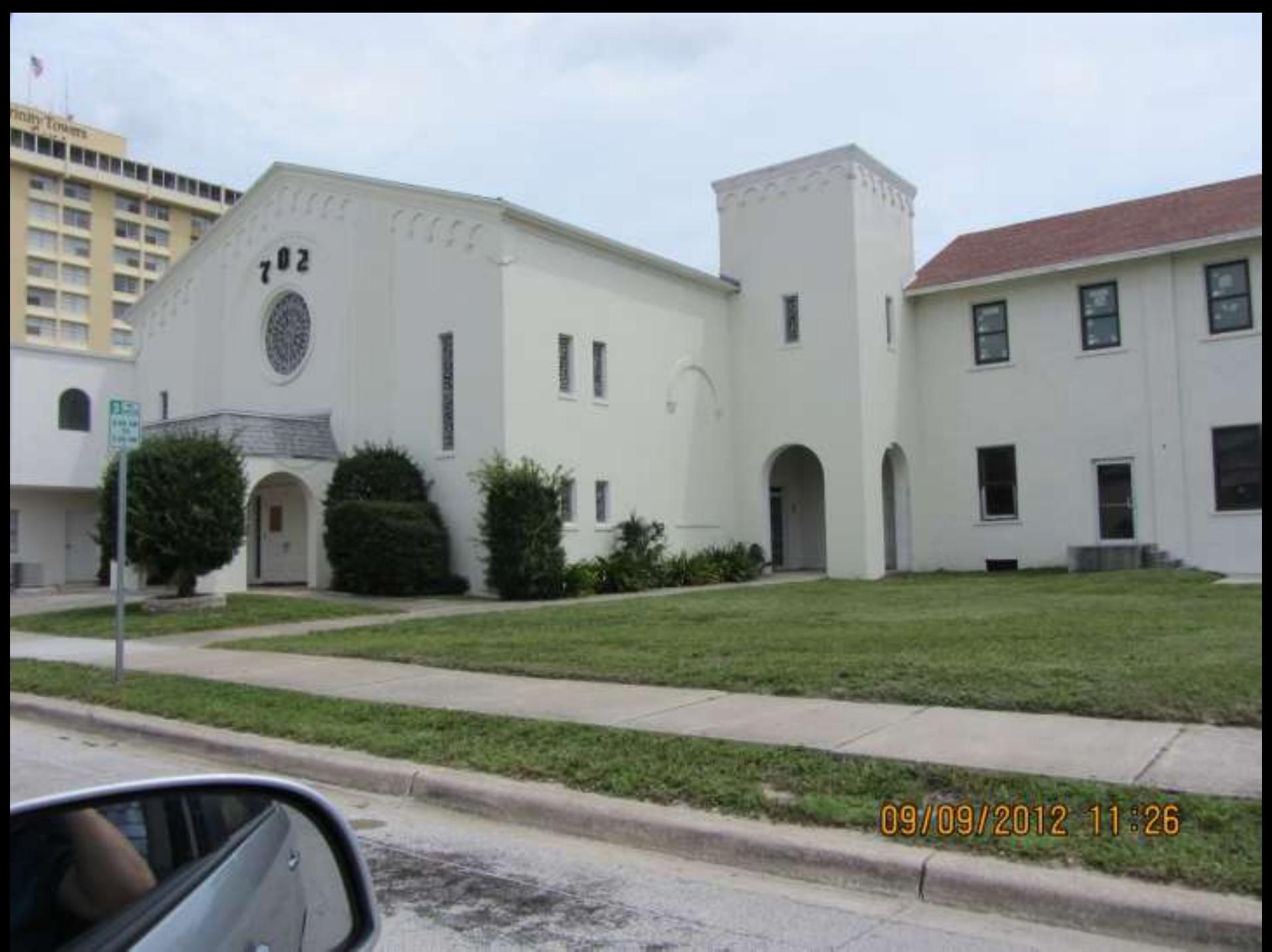




\section{Perform An Operator Needs Assessment}

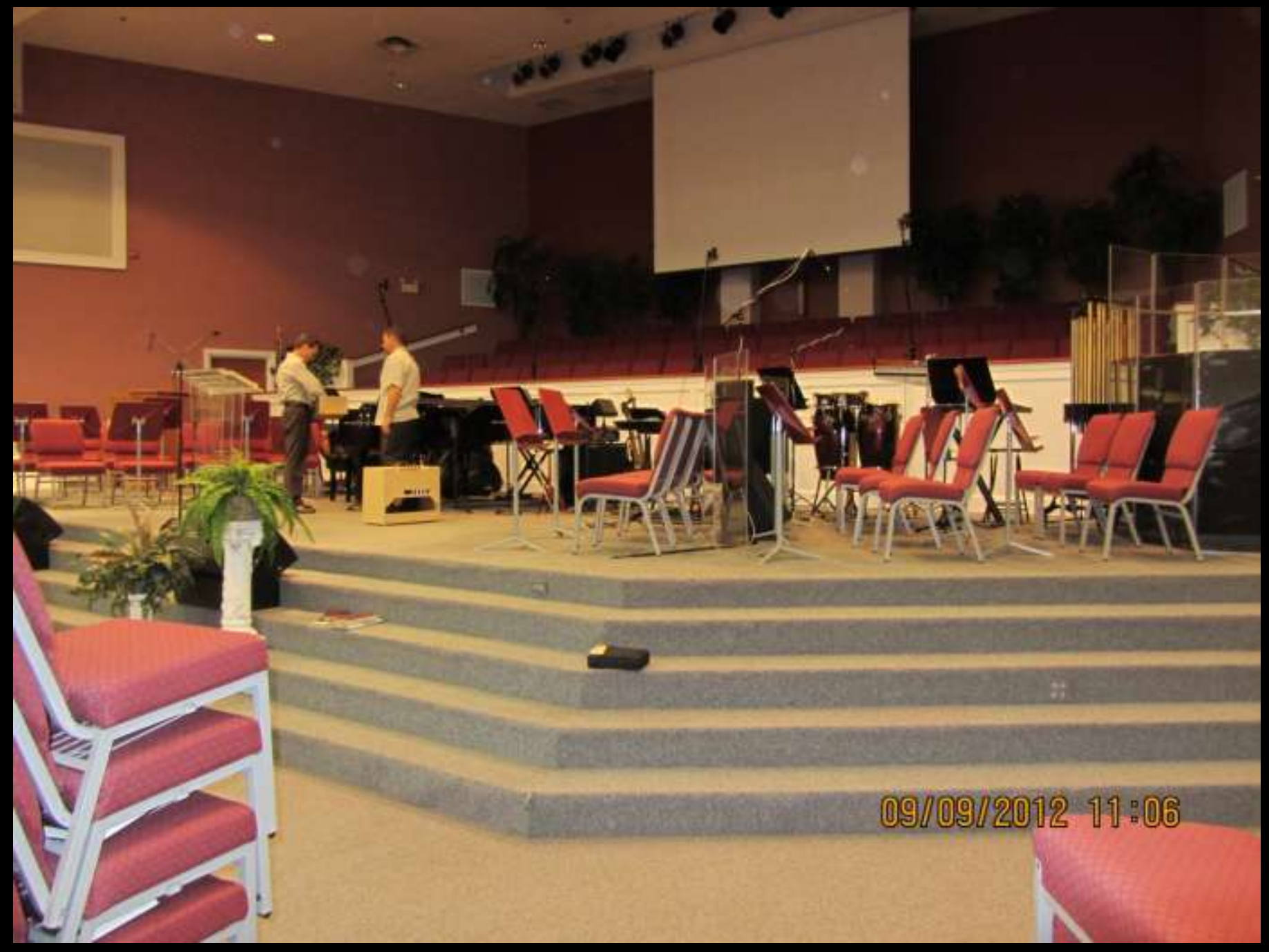




\section{Perform An Operator Needs Assessment}

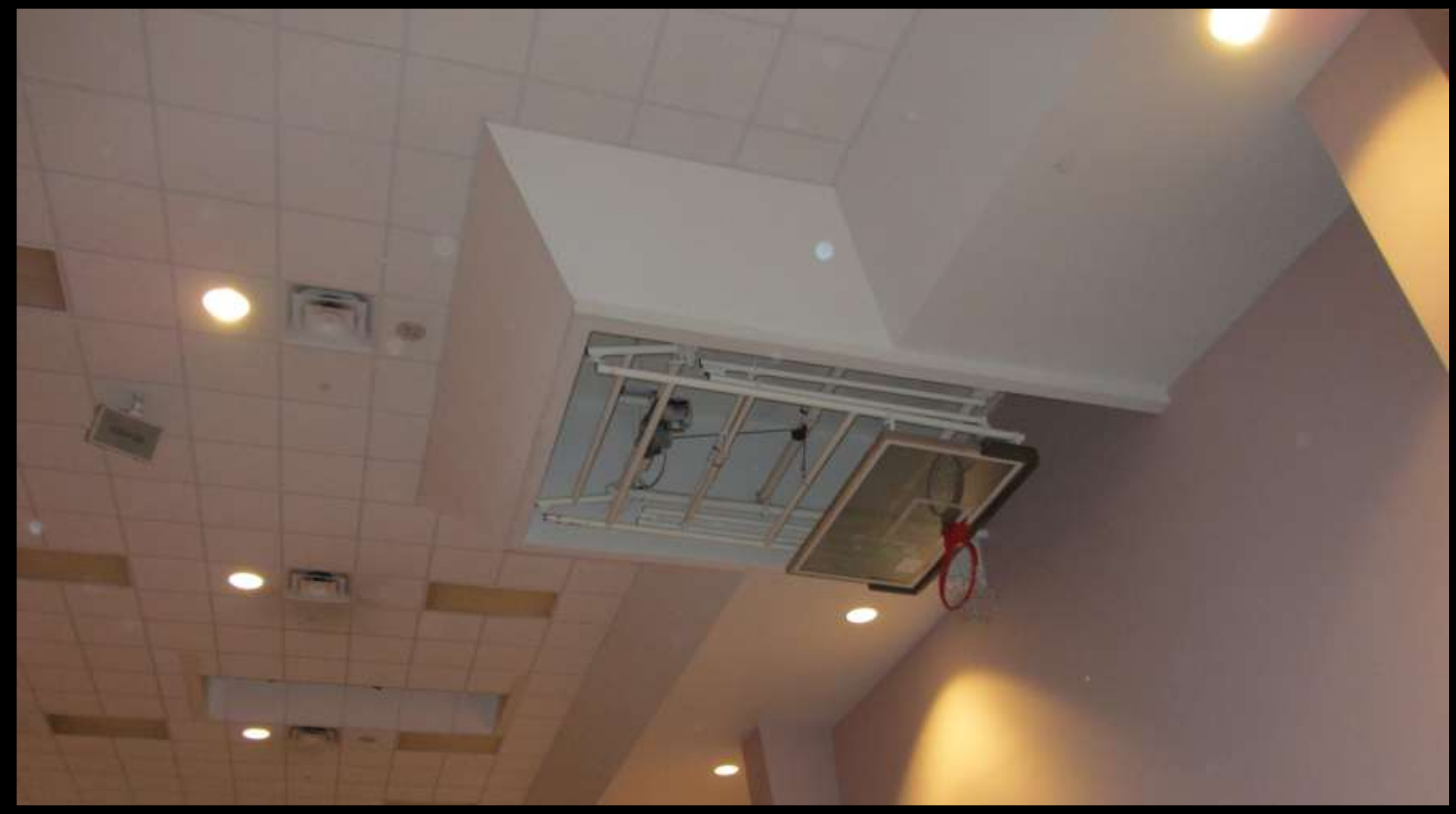


Perform An Operator Needs Assessment 


\section{Perform An Operator Needs}

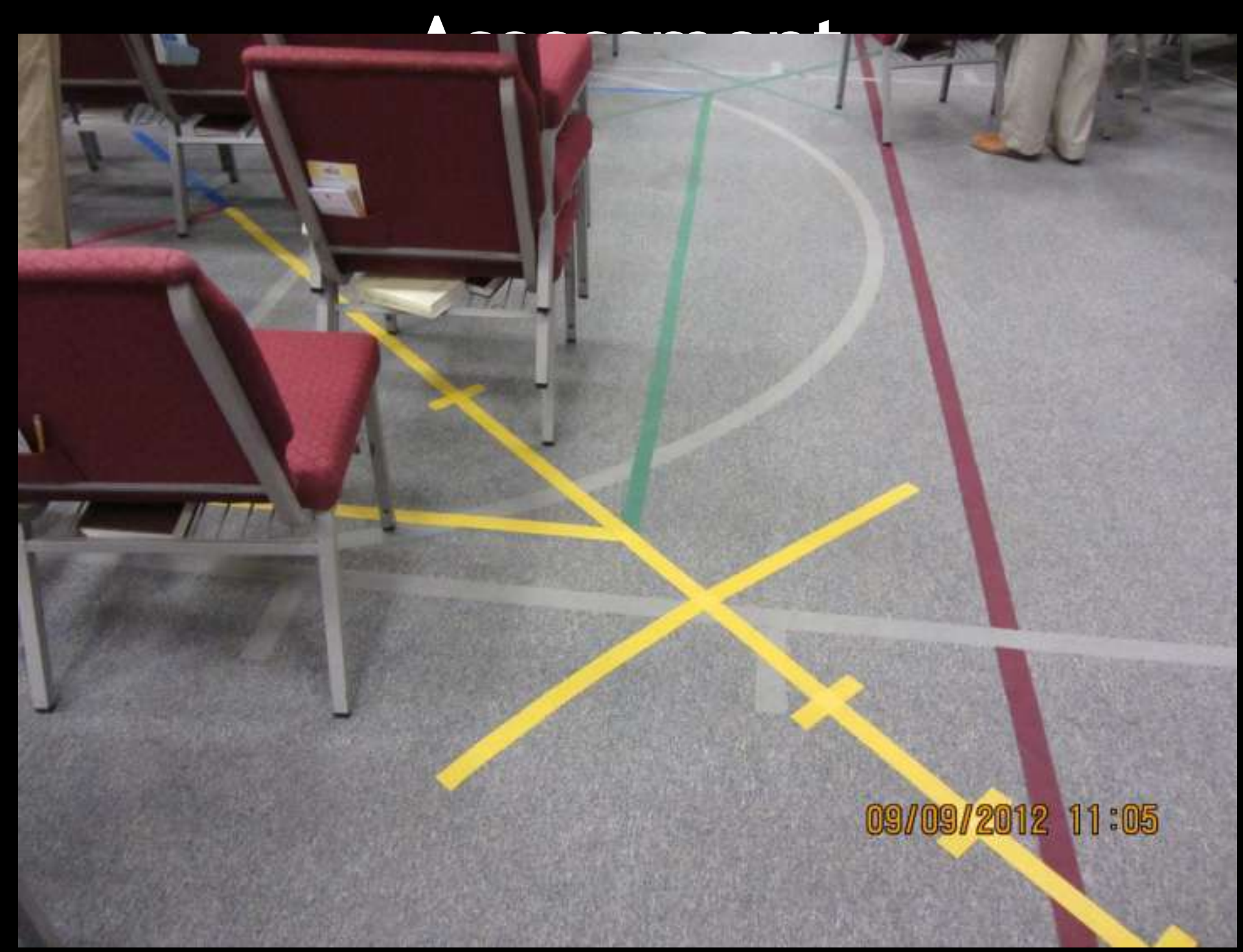


Perform An Operator Needs

Assessment

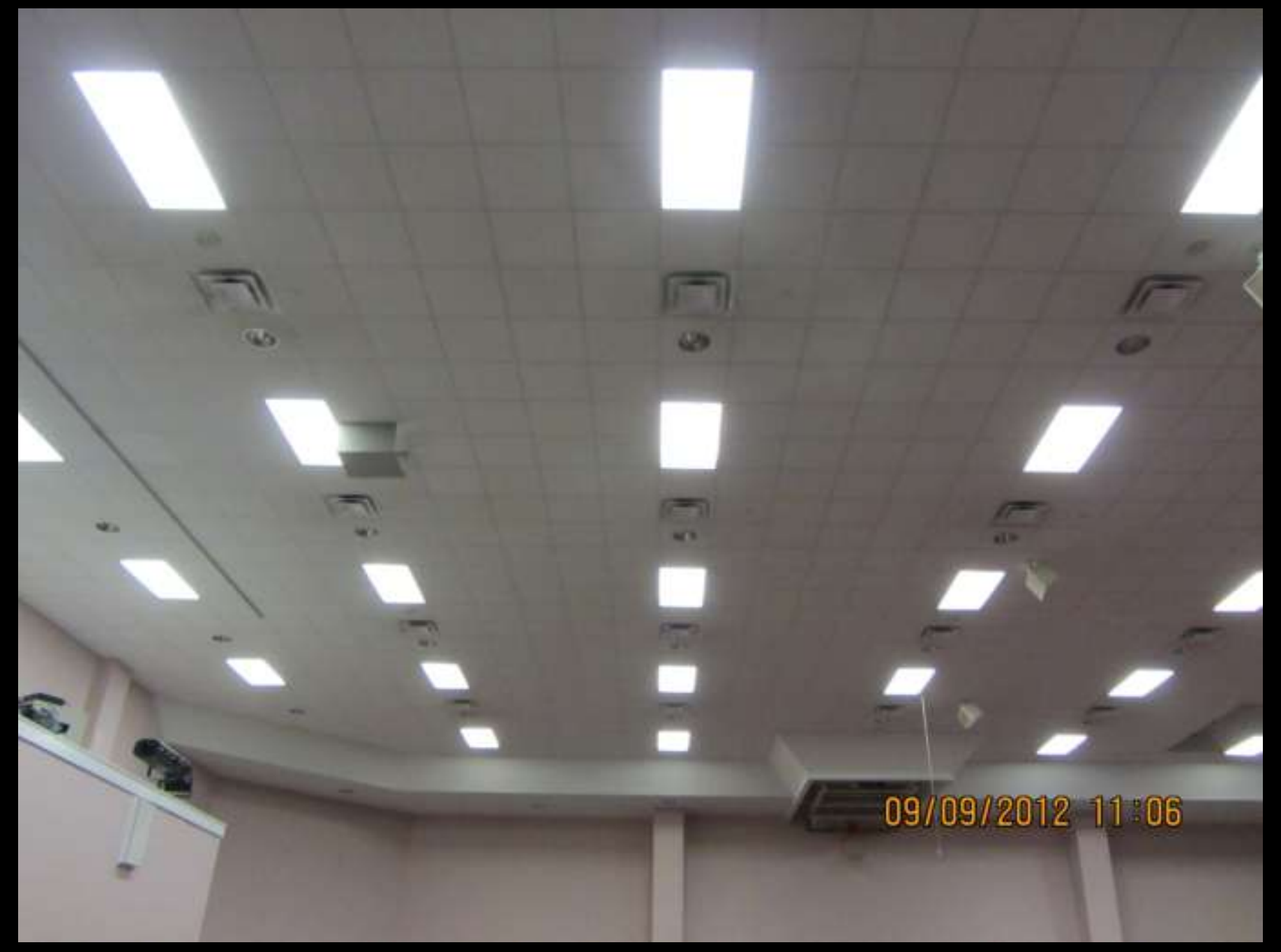




\section{Information}

\begin{tabular}{|l|l|l|}
\hline \multicolumn{1}{|c|}{ Important Information } & \multicolumn{1}{c|}{ Unimportant Information } \\
\hline User & Facility time requirements & Detailed operations schedule \\
\hline Owner & Facility availability & Other user schedules \\
\hline User & Office space requirements & Management team identification \\
\hline Owner & Undocumented changes & Last occupant \\
\hline User & Spacecraft hazardous propellants & Orbital Parameters \\
\hline
\end{tabular}


Survey - Problems 


\section{Analyze The Facility's Capabilities, Capacity, And Restrictions}

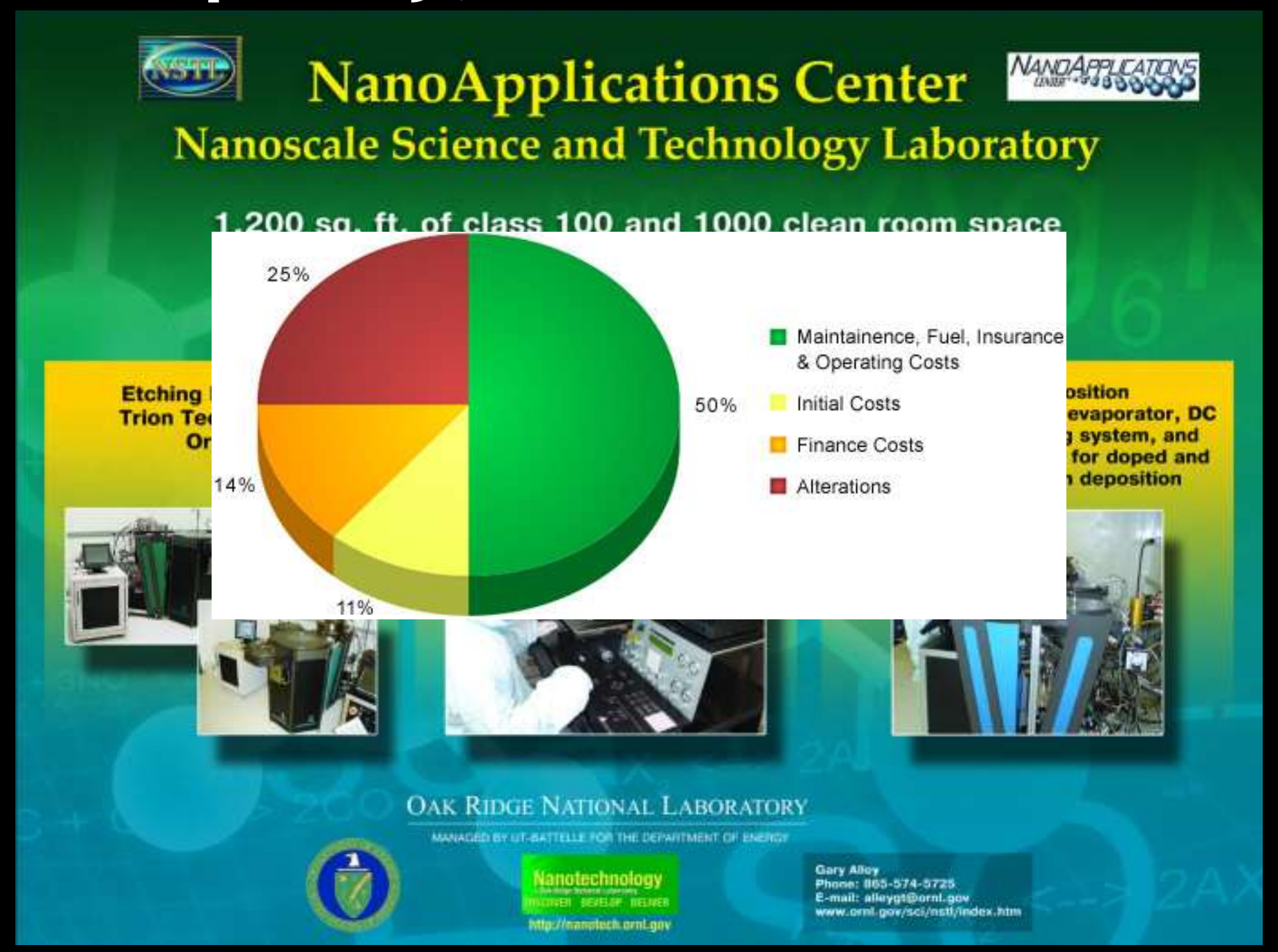




\section{Perform A Gap Analysis}

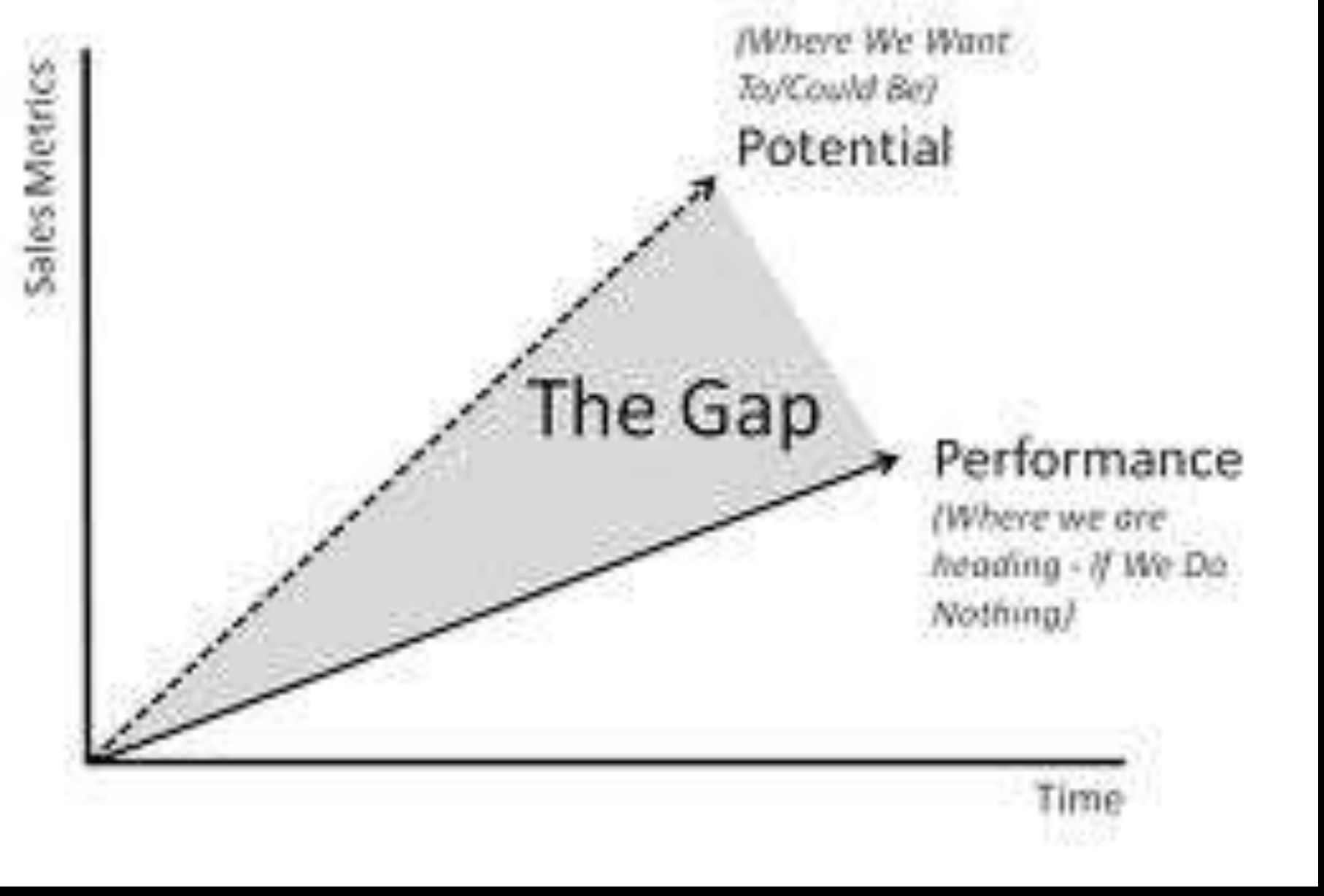




\section{Develop Operational Concepts}

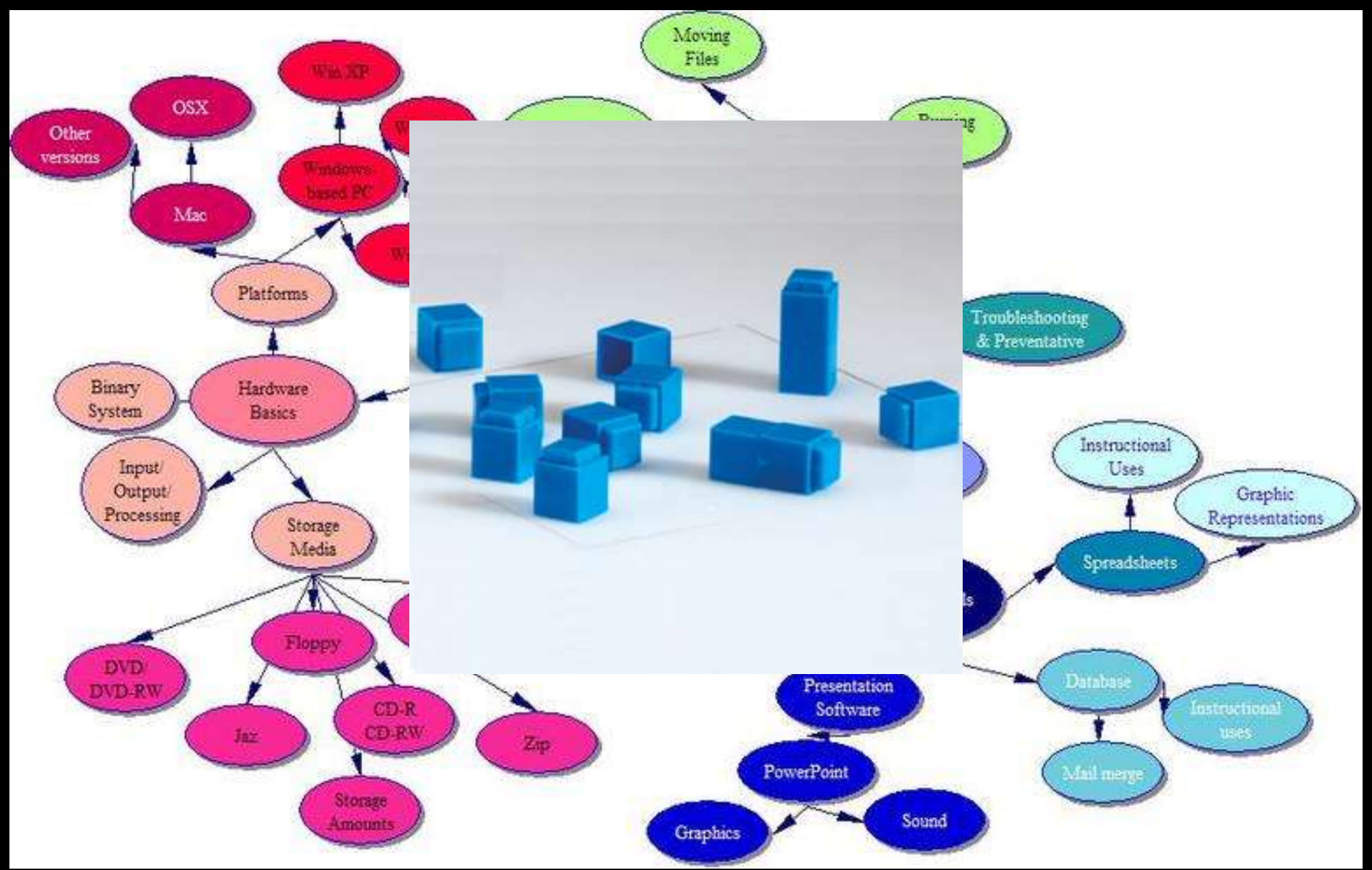




\section{Each Concept Should Include}

- An overall operational concept

- Constraints limiting the use of the facility

- Types of users for which the facility is suited

- Needed facility modifications

- Operational policies, and procedures required for multiple users 


\section{Map Modifications To Concepts}

\begin{tabular}{|c|}
\hline Modification Areas (per Concept) \\
Life Safety \\
\hline Fire Suppression \\
\hline Fire Alarm and Detection Systems \\
\hline Physical Security/Access Control \\
Visual Separation \\
\hline Safety/Debris Protection \\
\hline Mechanical Systems \\
\hline High Pressure Gas Systems \\
\hline Ground Coolant Systems \\
\hline HVAC Systems \\
\hline AHU and CHW/HHW Metering \\
\hline ML/MLP High Pressure Gas interfaces \\
\hline Electrical Systems \\
\hline Electrical Systems Metering \\
\hline IT/Communications \\
\hline Power Systems Reliability \\
\hline Operational Policies and Considerations \\
\hline Operation Control Locations \\
\hline
\end{tabular}




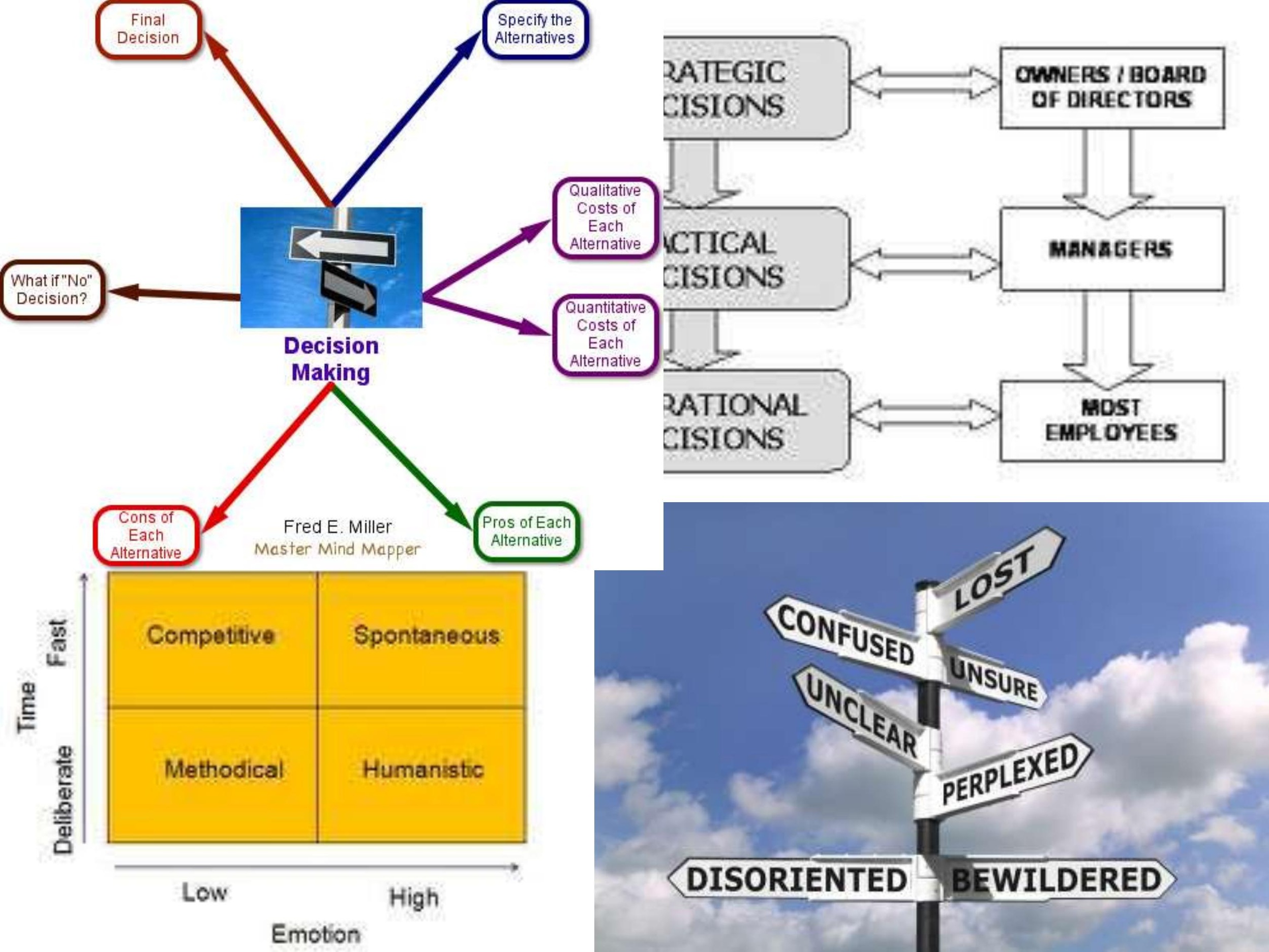




\section{Make Decisions}

The modification list can be extensive. The owner should use whatever decision process best suits his organization, however the allowed types of decisions should be a controlled set. The process is highly iterative and it is easy to get lost in that process. 


\section{Allowable Decisions}

- Do the change

- Do the change when customer appears

- Expect the customer to provide

- Never do the change

- Neutral - make no decision until forced to do so 


\section{Multi-Use O\&C}

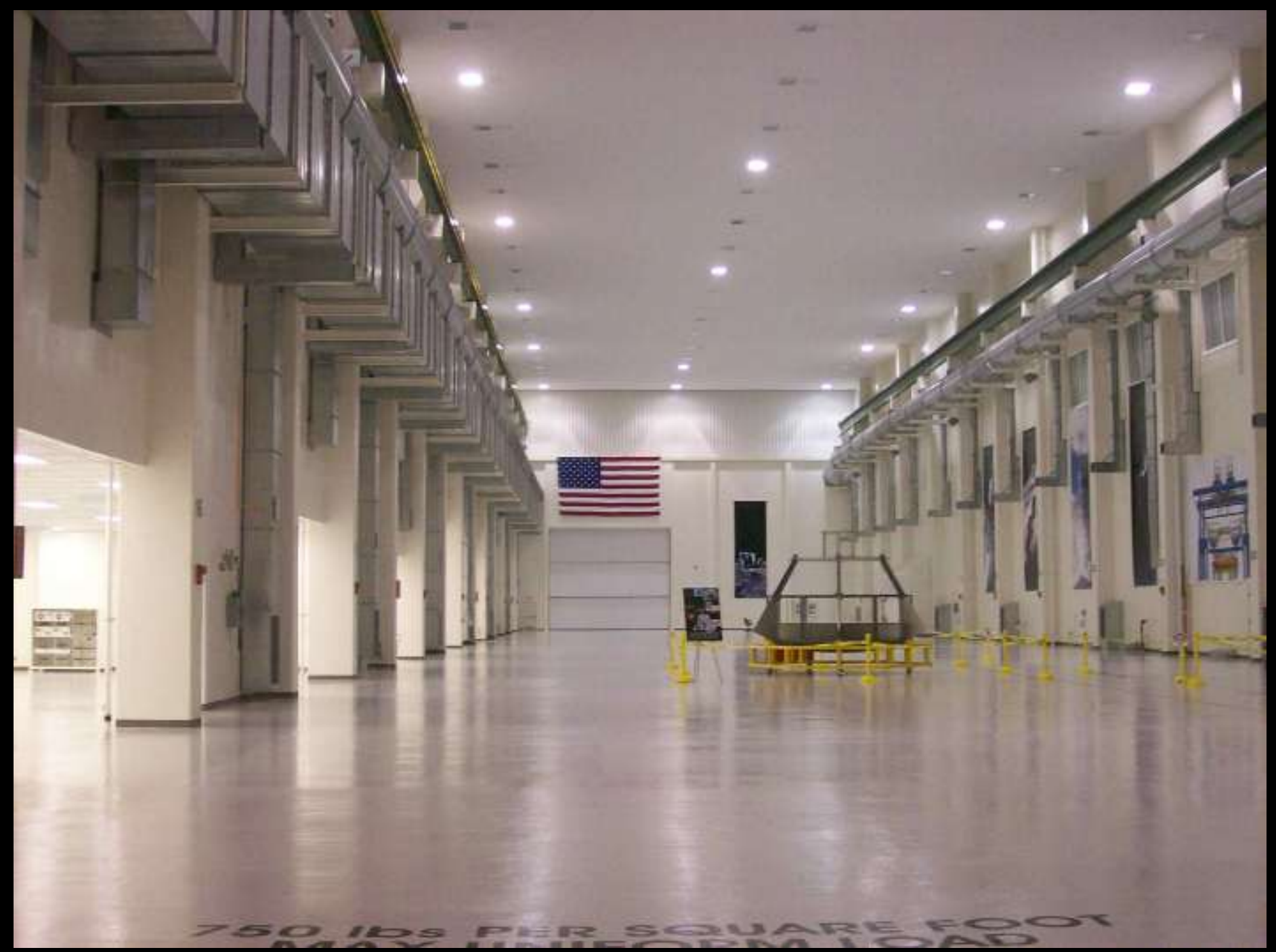




\section{When starting such a process consider:}

- Organically grown systems not conducive to isolation, metering, etc.

- Cost to upgrade existing facilities (e.g. Code, Infrastructure) can be very high

- Expectations of future occupancy are generally unrealistically high

- Be prepared for unexpected cost drivers, e.g. life safety access

- Consider not only total users but also impact between users due to parallel operations

- Operations always take longer than scheduled

- Have realistic expectations of when your facility will be available - existing tenants schedules may extend

- Modifications take longer than planned

- Recognize down time (unoccupied) period requirements due to modifications or maintenance

- Before modifying for parallel tenants, understand if money is available for parallel programs

- "World Class" may be mythical. It drives

Higher costs

Decreased flexibility

- Generally applicable only to a specific class of customers 


\section{When starting such a process consider (2):}

- Metering - is it really necessary?

- How much full cost accounting to each individual client is appropriate?

- Understand future maintenance costs and its impact to customers

- Recognize the down time for implementing multi-use. Single use may be appropriate.

- Technical vs. Policy Decisions - know which ones you are making

- What does your anchor customer want and how do you keep him happy above all others?

- Identify Tenant vs. Common Areas

- Who pays?

- Know current and future operational restrictions

- Recognize your tenants safety and proprietary information concerns

- Beware of upgrade for upgrade sake

- Know what tenants will bring with them - it may reduce your costs 


\section{Biggest Lesson}

The Best Multi-use modification process

Be Multi-use from the beginning 
What Do You Want To Be When You Grow Up? 


\section{What Do You Want To Be When You Grow Up?}

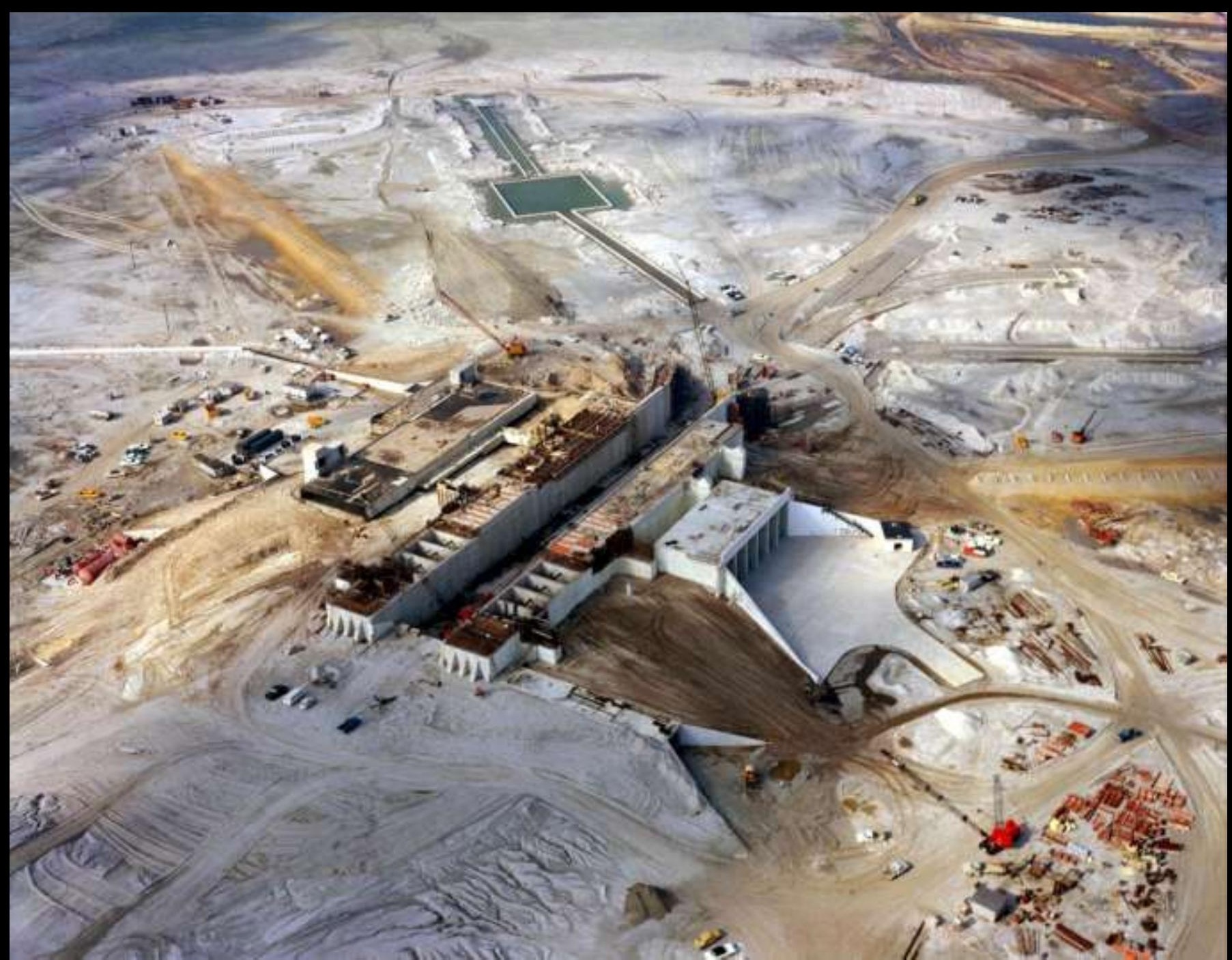




\section{Steps In The Process}

- Understand why multi-use is desirable

- Decide the best usage categories

- Identify target tenants

- Perform an operator needs assessment

- Analyze the facility's capabilities, capacity, and restrictions

- Perform a gap analysis

- Develop Operational Concepts

- Map modifications to concepts

- Make decisions 


\section{Do Not Get Lost In the Process}

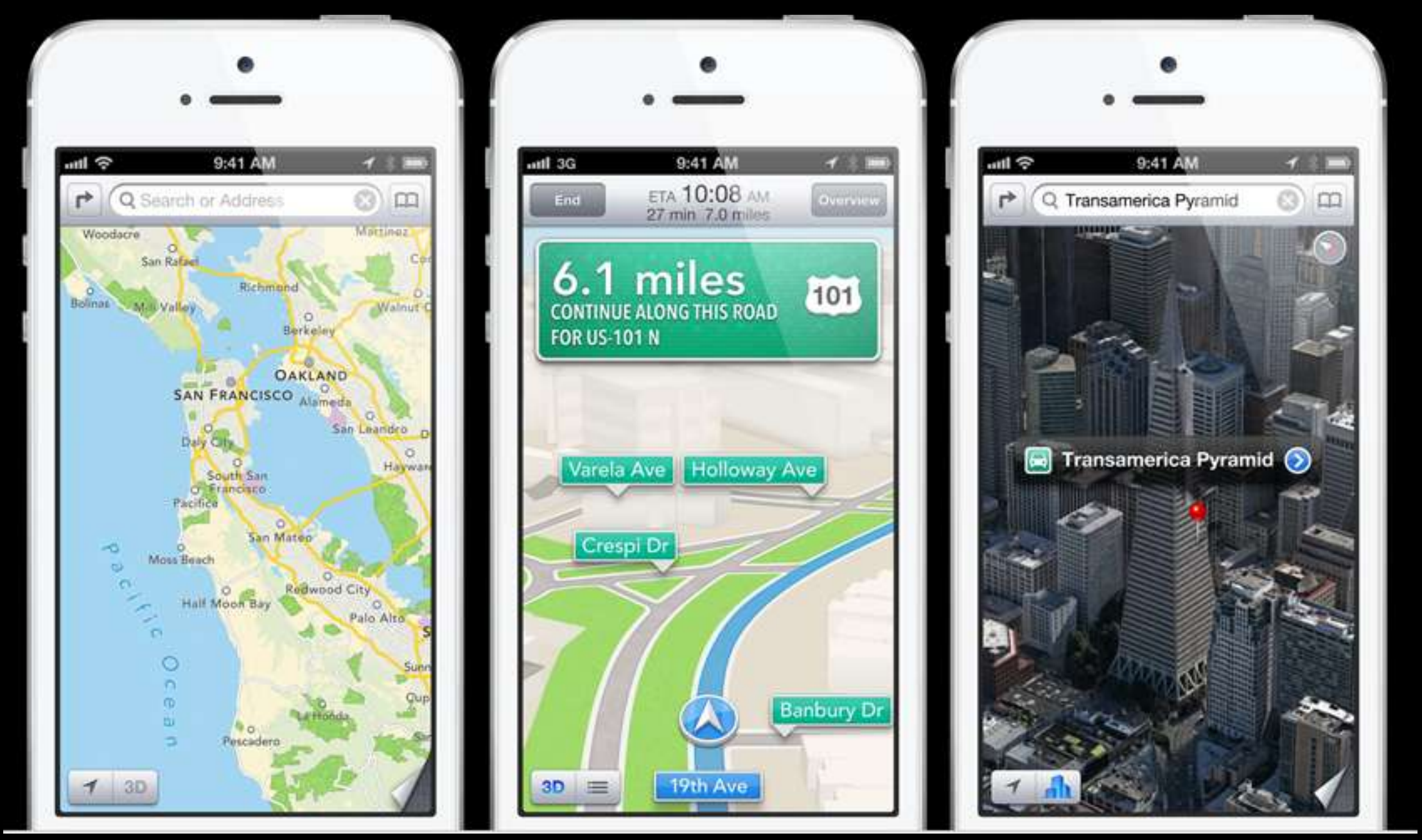




\section{Alan T. DeLuna}

Dave Hertzler

Multi-Use Facilities - Repurposing

Facilities Infrastructure to Support the Multi-customer Environment 